\title{
ON ERROR ESTIMATES FOR GALERKIN FINITE ELEMENT METHODS FOR THE CAMASSA-HOLM EQUATION
}

\author{
D.C. ANTONOPOULOS, V. A. DOUGALIS, AND DIMITRIOS MITSOTAKIS
}

\begin{abstract}
We consider the Camassa-Holm $(\mathrm{CH})$ equation, a nonlinear dispersive wave equation that models one-way propagation of long waves of moderately small amplitude. We discretize in space the periodic initial-value problem for $\mathrm{CH}$ (written in its original and in system form), using the standard Galerkin finite element method with smooth splines on a uniform mesh, and prove optimal-order $L^{2}$-error estimates for the semidiscrete approximation. We also consider an initial-boundary-value problem on a finite interval for the system form of $\mathrm{CH}$ and analyze the convergence of its standard Galerkin semidiscretization. Using the fourth-order accurate, explicit, "classical" Runge-Kutta scheme for time-stepping, we construct a highly accurate, stable, fully discrete scheme that we employ in numerical experiments to approximate solutions of $\mathrm{CH}$, mainly smooth travelling waves and nonsmooth solitons of the 'peakon' type.
\end{abstract}

\section{INTRODUCTION}

In this paper we analyze standard Galerkin finite element approximations to the Camassa-Holm $(\mathrm{CH})$ equation

$$
u_{t}+2 k u_{x}+3 u u_{x}-u_{x x t}=2 u_{x} u_{x x}+u u_{x x x} .
$$

The equation may be derived as a bi-Hamiltonian integrable system by the method of [26] (cf. 24], 25]). It is named after R. Camassa and D.D. Holm, who derived it in 7 from the Euler equations of water-wave theory as a model for the unidirectional propagation of long waves on the surface of an ideal fluid in a uniform horizontal channel. In (1.1), which is written in nondimensional, unscaled variables, $u=u(x, t)$ is proportional to the depth-averaged horizontal velocity of the fluid at $x, t$, that are proportional to position along the channel and time, respectively. The equation was further studied from this viewpoint in 29], and was rigorously justified in [17] as an $O\left(\sigma^{4}\right)$-accurate unidirectional approximation to the Euler equations when the scaling parameters satisfy $\sigma \ll 1, \epsilon=O(\sigma)$. Here $\epsilon=a / h_{0}, \sigma^{2}=h_{0}^{2} / \lambda^{2}, h_{0}$ is the depth of the channel, $a$ is a typical surface wave amplitude, and $\lambda$ a typical wavelength. In scaled, nondimensional variables such a derivation leads e.g. to the equation

$$
v_{\tau}+v_{y}+\epsilon v v_{y}-\sigma^{2} v_{y y \tau}-\frac{\epsilon \sigma^{2}}{3}\left(2 v_{y} v_{y y}+v v_{y y y}\right)=O\left(\sigma^{4}\right),
$$

from which we may recover (1.1) by replacing the right-hand side by zero and making the change of variables $u(x, t)=2 k \epsilon v(y, \tau) / 3, y=\sigma x, \tau=2 k \sigma t$. Hence, when compared with the scaled BBM equation, for which $\sigma \ll 1, \epsilon=O\left(\sigma^{2}\right)$, the equation (1.2) approximates the Euler equations to the same order of formal accuracy, possesses an additional nonlinear dispersive term, and remains valid in the larger wave-amplitude regime $\epsilon=O(\sigma)$.

The Camassa-Holm equation has been studied extensively and shown to possess global smooth solutions, but also solutions that develop 'breaking wave' singularities in finite time; in such singular solutions the derivative $u_{x}$ blows up while $u$ remains bounded. As expected from its complete integrability properties, the equation possesses solitons, i.e. solitary waves that interact cleanly. It should be noted that (1.1) may be written in system form e.g. as

$$
\begin{aligned}
& m=u-u_{x x}, \\
& m_{t}+2 k u_{x}+u m_{x}+2 u_{x} m=0 .
\end{aligned}
$$

Date: April 2, 2019.

2010 Mathematics Subject Classification. 65M60, 35Q53.

Key words and phrases. Error estimates, Galerkin / Finite element method, Camassa-Holm equation, peakons. 
The literature on the well-posedness of the initial-value problem (ivp) for the $\mathrm{CH}$ is large; here we will just mention some basic references. (A survey of results up to 2003 may be found in [37. It should be noted that in some of the works to be mentioned below $k$ is taken equal to zero in (1.1) and in (1.3); for the significance of $k$ in the context of travelling-wave solutions, see the remarks below.) In [15. Constantin and Escher established the existence of local in time solutions in $H^{3}$ for (1.1) with $k=0$, and provided sufficient conditions on the initial data that lead to global solutions or to the emergence of singularities in finite time. Analogous results for the periodic ivp for the $\mathrm{CH}$, written in the system form (1.3) with $k=0$, were established in [13] in the Sobolev space $H_{p e r}^{2}$ of periodic functions. In [35] Li and Olver studied the well-posedness of the ivp for a slightly generalized form of (1.1) in $H^{s}$ for $s>3 / 2$, put forth alternative conditions to those of 15 for global existence and finite-time blow up of solutions, and also studied the existence of weaker solutions in $H^{s}$ for $1<s \leq 3 / 2$. The development of singularities has been studied in several papers in addition to those already mentioned, e.g. in [16], [37, and in [17; in the latter reference the large-time $(O(T / \epsilon))$ existence of solutions and the breaking of waves of the free surface equation (in addition to the equation of the velocity $u$ ) have also been analyzed. More recently, issues of existence of global generalized solutions, possibly after wave-breaking occurs, have been studied, cf. e.g. 6]. We also note that the well-posedness of initial-boundary value problems (ibvp's) for $\mathrm{CH}$ (usually written in the system form (1.3) ) has also been studied; see e.g. [33] for the local well-posedness in $H^{4}$ of the ibvp for (1.3) with $k=0$ on a finite interval with boundary conditions $u=m=0$ at the endpoints, and also [23], where the well-posedness of the quarter-plane and the two-point ibvp has been studied in more general spaces.

Since CH is completely integrable it is amenable to study in terms of the Inverse Scattering Transform (IST), cf. e.g. [14], [12, and their references. A complete classification of its travelling-wave solutions has been carried out in 34; such solutions may be smooth or nonsmooth, in the latter case satisfying the equation in the sense of distributions. Its solitary waves are solitons. If $k \neq 0 \mathrm{CH}$ possesses smooth solitons and N-solitons; these have been explicitly constructed, and their interactions studied by IST; cf. [30], and especially [38, 39, 40, 41. For $k=0$ the equation does not have smooth solitary waves; it has however generalized soliton solutions ('peakons') of the form $u(x, t)=c \exp (-|x-c t|)$ for any $c>0$; the study of existence and interactions of peakons has attracted a lot of attention, starting with [7]. (As previously mentioned, other types of singular travelling-wave solutions have been identified in [34.) Finally, let us mention that the stability of smooth solitons and peakons has been established in [19, [20]; see also [18].

A note on the constant $k$ that appears in (1.1): As the change of variables that connects the scaled version (1.2) of $\mathrm{CH}$ with (1.1) implies, $k$ is related to the speed of e.g. the travelling-wave solutions of (1.2), and, therefore, for physical water waves it is necessary that $k \neq 0$. We may assume that $k>0$, since, otherwise, the change of variables $U(X, T)=-u(x, t), X=-x, T=t$ leads to a $\mathrm{CH}$ equation with $-2 k U_{X}$ instead of $2 k u_{x}$. It has also been noticed since [7] that the change of variables $w(z, s)=u(x, t)+k, z=x+k t, s=t$, transforms (1.1) into

$$
w_{s}+3 w w_{z}-w_{z z s}=2 w_{z} w_{z z}+w w_{z z z}
$$

i.e. the $\mathrm{CH}$ without the $2 k u_{x}$ term. (We will usually write (1.4) using the variables $u, x, t$ and call it, cf. [38, reduced $\mathrm{CH}$ equation ( $\mathrm{RCH})$.) As a consequence of this transformation and previous remarks on the existence of solitary waves of $\mathrm{CH}$ and $\mathrm{RCH}$, it follows that $\mathrm{RCH}$ possesses smooth travelling-wave solutions decaying at infinity to $k$, and $\mathrm{CH}$ with $k \neq 0$ possesses peakon-type travelling waves decaying to $-k$.

Since the derivation of $\mathrm{CH}$, numerical methods have been used in an exploratory fashion to illuminate aspects of the generation and interactions of its solutions, see e.g. [8]. The ensuing numerical literature is large; we confine ourselves to mentioning a few papers that the reader may consult along with their references. Among works that focus on the analysis of numerical methods and error estimates cf. e.g. [32] (spectral methods), 28] and [1] (finite difference methods), and 9] (particle methods). Works that focus on the construction of numerical schemes and numerical experimentation include e.g. 31] (spectral methods), [46] and [36] (DG methods), and [10] (particle methods).

In the paper at hand we consider Galerkin finite element methods for the numerical solution of $\mathrm{CH}$. In Sections 2 and 3 we provide the necessary background and notation and prove optimal-order-of-convergence $L^{2}$-error estimates for the standard Galerkin semidiscrete approximation for the periodic ivp for (1.1) using smooth splines on a uniform mesh. The proof is effected by comparing the semidiscrete approximation with the Thomée-Wendroff quasiinterpolant, 45]. In Section 4 we consider the analogous periodic ivp for 
the system (1.3) and prove $L^{2}$-error estimates of optimal rate of convergence for the semidiscrete standard Galerkin approximations of $m$ and $u$ using again smooth periodic splines on a uniform mesh and employing similar error estimation techniques. We refer to this numerical scheme as the 'modified' Galerkin method and note that it requires splines of order $r \geq 2$ (i.e. continuous piecewise polynomial functions of degree $r-1 \geq 1$ ), thus allowing the use of piecewise linear continuous functions, as opposed to the standard Galerkin scheme for (1.1) that requires $r \geq 3$, i.e. using at least $C^{1}$ quadratics due to the presence of the $u u_{x x x}$ term. In Section 5 we consider the ibvp for the system (1.3) on a finite interval with zero Dirichlet boundary conditions for $m$ and $u$ at the endpoints. We study the convergence of the semidiscrete approximation of the problem by the standard Galerkin method on a quasiuniform mesh with piecewise polynomial functions of degree $r-1$ with $r \geq 2$. Using energy estimates, properties of the $H^{1}$ - and $L^{2}$-projections onto the finite element spaces and duality techniques we prove a suboptimal $L^{2}$-error estimate of $O\left(h^{r-1}\right)$ for the approximation of $m$, and an optimal $H^{1}$-estimate of $O\left(h^{r}\right)$ and a suboptimal $L^{2}$-estimate of $O\left(h^{r-1 / 2}\right)$ for the approximation of $u$. We took $k=0$ for simplicity in Sections 3 3 , the inclusion of the $2 k u_{x}$ term poses no difficulty and the results are the same.

In Section 6 we present the results of some numerical experiments that we performed using the Galerkin methods described above in space and discretizing the ivp's for the attendant ode systems in the temporal variable by the classical, explicit, fourth-order-accurate Runge-Kutta scheme. The resulting fully discrete methods are stable under a mild restriction on the Courant number $\Delta t / h$, as the problem is not very stiff due to the presence of the BBM type term $-u_{x x t}$ in the left-hand side of (1.1). In the numerical experiments we check the spatial order of convergence of the schemes in the case of smooth solutions and investigate experimentally the order of convergence for peakons. We check the conservation properties of the discrete schemes and study the fidelity of the numerical approximations of the travelling waves by computing their amplitude, phase, shape, and speed errors. Finally, we study the generation and interactions of peakons and compare the accuracy of our schemes with that of other numerical methods in the literature.

In this paper we denote, for integer $k \geq 0$, by $H^{k}=H^{k}(0,1)$ the usual $L^{2}$-based Sobolev spaces on $[0,1]$ by $\stackrel{\circ}{H}^{1}$ the subspace of $H^{1}$ consisting of functions with zero boundary conditions, and by $H_{\text {per }}^{k}$ the subspace of $H^{k}$ consisting of 1-periodic functions; in all cases the corresponding norms are denoted by $\|\cdot\|_{k}$. We let $C^{k}$ denote the $k$-times continuously differentiable functions on $[0,1]$ and $C_{p e r}^{k}$ the 1-periodic such functions. The inner product on $L^{2}=L^{2}(0,1)$ is denoted by $(\cdot, \cdot)$ and the corresponding norm simply by $\|\cdot\|$. The norms of $W_{\infty}^{k}=W_{\infty}^{k}(0,1)$ and $L^{\infty}=L^{\infty}(0,1)$ are denoted by $\|\cdot\|_{k, \infty}$ and $\|\cdot\|_{\infty}$, respectively. For a Banach space $X, C([0, T] ; X)$ denotes as usual the continuous maps from $[0, T]$ into $X . \mathbb{P}_{r}$ are the polynomials of degree at most $r$.

\section{Acknowledgements}

The authors would like to thank Mr. Gregory Kounadis for the numerical experiments in the case of the ibvp (CH-s-b). V.A.D. and D.E.M. acknowledge travel support by grant MTM2014-54710 of the Ministerió de Economia y Competividad, Spain. D.E.M. was supported by the Marsden Fund administered by the Royal Society of New Zealand with contract number VUW1418.

\section{Periodic Splines and the QUasinnterpolant}

As we will be interested in approximating solutions of the $\mathrm{CH}$ that are 1-periodic functions in the spatial variable, we let $N$ be a positive integer and $h=1 / N, x_{i}=i h, i=0,1, \ldots, N$, and for integer $r \geq 2$ consider the associated $N$-dimensional space of smooth 1 -periodic splines

$$
S_{h}=\left\{\phi \in C_{\text {per }}^{r-2}[0,1]:\left.\phi\right|_{\left[x_{i-1}, x_{i}\right]} \in \mathbb{P}_{r-1}, 1 \leq i \leq N\right\} .
$$

It is well known that $S_{h}$ has the following approximation properties: Given a sufficiently smooth 1-periodic function $v$, there exists $\chi \in S_{h}$ such that

$$
\sum_{j=0}^{s-1} h^{j}\|v-\chi\|_{j} \leq C h^{s}\|v\|_{s}, \quad 1 \leq s \leq r,
$$


and

$$
\sum_{j=0}^{s-1} h^{j}\|v-\chi\|_{j, \infty} \leq C h^{s}\|v\|_{s, \infty}, \quad 1 \leq s \leq r,
$$

for some constant $C$ independent of $h$ and $v$. Moreover, there exists a constant $C$ independent of $h$ such that the inverse properties

$$
\begin{aligned}
\|\chi\|_{\beta} \leq C h^{-(\beta-\alpha)}\|\chi\|_{\alpha}, & 0 \leq \alpha \leq \beta \leq r-1, \\
\|\chi\|_{s, \infty} \leq C h^{-(s+1 / 2)}\|\chi\|, & 0 \leq s \leq r-1,
\end{aligned}
$$

hold for all $\chi \in S_{h}$. (In the sequel we shall denote by $C$ generic constants independent of $h$.)

Thomée and Wendroff, [45], proved that there exists a basis $\left\{\phi_{j}\right\}_{j=1}^{N}$ of $S_{h}$ with $\operatorname{supp}\left(\phi_{j}\right)=O(h)$, such that if $v$ a sufficiently smooth 1-periodic function, the associated quasiinterpolant $Q_{h} v=\sum_{j=1}^{N} v\left(x_{j}\right) \phi_{j}$ satisfies

$$
\left\|Q_{h} v-v\right\| \leq C h^{r}\left\|v^{(r)}\right\| .
$$

In addition, they showed that the basis $\left\{\phi_{j}\right\}_{j=1}^{N}$ may be chosen so that the following properties hold:

(i) If $\psi \in S_{h}$, then

$$
\|\psi\| \leq C h^{-1} \max _{1 \leq i \leq N}\left|\left(\psi, \phi_{i}\right)\right| .
$$

(It follows from (2.2) that if $\psi \in S_{h}, f \in L^{2}$ are such that

$$
\left(\psi, \phi_{i}\right)=\left(f, \phi_{i}\right)+O\left(h^{\alpha}\right), \quad \text { for } \quad 1 \leq i \leq N,
$$

then $\left.\|\psi\| \leq C h^{\alpha-1}+\|f\|.\right)$

(ii) Let $w$ be a sufficiently smooth 1-periodic function and $\nu, \kappa$ integers such that $0 \leq \nu, \kappa \leq r-1$. Then

$$
\left(\left(Q_{h} w\right)^{(\nu)}, \phi_{i}^{(\kappa)}\right)=(-1)^{\kappa} h w^{(\nu+\kappa)}\left(x_{i}\right)+O\left(h^{2 r+j-\nu-\kappa}\right), \quad 1 \leq i \leq N,
$$

where $j=1$ if $\nu+\kappa$ is even and $j=2$ if $\nu+\kappa$ is odd.

(iii) Let $f, g$ be sufficiently smooth 1-periodic functions and $\nu$ and $\kappa$ as in (ii) above. Let

$$
\beta_{i}=\left(f\left(Q_{h} g\right)^{(\nu)}, \phi_{i}^{(\kappa)}\right)-(-1)^{\kappa}\left(Q_{h}\left[\left(f g^{(\nu)}\right)^{(\kappa)}\right], \phi_{i}\right), \quad 1 \leq i \leq N .
$$

Then

$$
\max _{1 \leq i \leq N}\left|\beta_{i}\right|=O\left(h^{2 r+j-\nu-\kappa}\right)
$$

where $j$ as in (ii).

It follows from the approximation and inverse properties of $S_{h}$, and (2.1) that if $r \geq 2, v \in H_{p e r}^{r}(0,1) \cap$ $W_{\infty}^{r}(0,1)$ and $V=Q_{h} v$, then

$$
\begin{aligned}
& \|V-v\|_{j} \leq C h^{r-j}\|v\|_{r}, j=0,1,2, \text { if } r \geq 3, j=0,1, \text { if } r=2, \\
& \|V-v\|_{j, \infty} \leq C h^{r-j-1 / 2}\|v\|_{r, \infty}, j=0,1,2, \text { if } r \geq 3, j=0,1, \text { if } r=2, \\
& \|V\|_{j} \leq C, \text { and }\|V\|_{j, \infty} \leq C, j=0,1,2, \text { if } r \geq 3, j=0,1, \text { if } r=2 .
\end{aligned}
$$

(The inequalities (2.7) follow from (2.5) and (2.6).)

\section{Standard Galerkin Semidiscretization of the PERIodic PROBlem}

In this section we consider the periodic initial-value problem for the Camassa-Holm equation in its reduced form. For $0 \leq t \leq T$ we seek $u=u(x, t), 1$-periodic in $x$ and satisfying

(C-H-per)

$$
\begin{aligned}
& u_{t}-u_{t x x}+3 u u_{x}=2 u_{x} u_{x x}+u u_{x x x}, \quad 0 \leq x \leq 1, \quad 0 \leq t \leq T, \\
& u(x, 0)=u_{0}(x), \quad 0 \leq x \leq 1 .
\end{aligned}
$$

Constantin, [13], showed that (C-H-per) is well-posed in $H_{p e r}^{2}$ on some temporal interval $[0, T], T=T\left(u_{0}\right)$. Here it will be assumed that (C-H-per) has a unique solution that is sufficiently smooth for the purposes of the error estimation. 
In the sequel we will denote the $H^{1}$ inner product by $A(\phi, \chi)=(\phi, \chi)+\left(\phi^{\prime}, \chi^{\prime}\right), \quad \phi, \chi \in H_{p e r}^{1}$. In addition we note that the right-hand side of the pde in (C-H-per) may be written as

$$
2 u_{x} u_{x x}+u u_{x x x}=u_{x} u_{x x}+\left(u u_{x x}\right)_{x}=\frac{1}{2}\left(u_{x}^{2}\right)_{x}+\left(u u_{x x}\right)_{x} .
$$

Taking this into account and using integration by parts we define the semidiscrete approximation $u_{h}$ of C-H-per in $S_{h}$ for $r \geq 3$ as the map $u_{h}:[0, T] \rightarrow S_{h}$ such that

$$
\begin{aligned}
& \left(u_{h t}, \phi\right)+\left(u_{h t x}, \phi^{\prime}\right)+3\left(u_{h} u_{h x}, \phi\right)+\frac{1}{2}\left(u_{h x}^{2}, \phi^{\prime}\right)+\left(u_{h} u_{h x x} . \phi^{\prime}\right)=0, \quad \forall \phi \in S_{h}, \quad 0 \leq t \leq T, \\
& u_{h}(0)=Q_{h} u_{0} .
\end{aligned}
$$

We start the error analysis of the semidiscretization (3.1)-(3.2) by establishing its consistency with (C-H-per):

Lemma 3.1. Let $u$, the solution of (C-H-per), be sufficiently smooth and suppose that $r \geq 3$. If $U=Q_{h} u$ and $\psi:[0, T] \rightarrow S_{h}$ is such that

$$
A(\psi, \phi)=\left(U_{t}, \phi\right)+\left(U_{t x}, \phi^{\prime}\right)+3\left(U U_{x}, \phi\right)+\frac{1}{2}\left(U_{x}^{2}, \phi^{\prime}\right)+\left(U U_{x x}, \phi^{\prime}\right), \quad \forall \phi \in S_{h},
$$

then, there exists a constant $C$ independent of $h$, such that

$$
\max _{0 \leq t \leq T}\|\psi(t)\|_{1} \leq C h^{r}
$$

Proof. Let $\rho=Q_{h} u-u=U-u$. Then

$$
A(\psi, \phi)=\left(\rho_{t}, \phi\right)+\left(\rho_{t x}, \phi^{\prime}\right)+3\left(U U_{x}-u u_{x}, \phi\right)+\frac{1}{2}\left(U_{x}^{2}-u_{x}^{2}, \phi^{\prime}\right)+\left(U U_{x x}-u u_{x x}, \phi^{\prime}\right), \quad \forall \phi \in S_{h} .
$$

Since

$$
\begin{aligned}
& U U_{x}-u u_{x}=(\rho+u)\left(\rho_{x}+u_{x}\right)-u u_{x}=\rho \rho_{x}+u_{x} \rho+u \rho_{x}, \\
& U_{x}^{2}-u_{x}^{2}=\rho_{x}\left(U_{x}+u_{x}\right)=\rho_{x}\left(\rho_{x}+2 u_{x}\right)=\rho_{x}^{2}+2 u_{x} \rho_{x}, \\
& U U_{x x}-u u_{x x}=(\rho+u)\left(\rho_{x x}+u_{x x}\right)-u u_{x x}=\rho \rho_{x x}+u_{x x} \rho+u \rho_{x x},
\end{aligned}
$$

we have from (3.5) that

$$
A(\psi, \phi)=\left(\rho_{t}, \phi\right)+3\left(\rho \rho_{x}+u_{x} \rho, \phi\right)+\frac{1}{2}\left(\rho_{x}^{2}, \phi^{\prime}\right)+\left(\rho \rho_{x x}+u_{x x} \rho, \phi^{\prime}\right)+(\omega, \phi), \quad \forall \phi \in S_{h},
$$

where $\omega:[0, T] \rightarrow S_{h}$ is defined by

$$
(\omega, \phi)=\left(\rho_{t x}, \phi^{\prime}\right)+3\left(u \rho_{x}, \phi\right)+\left(u_{x} \rho_{x}, \phi^{\prime}\right)+\left(u \rho_{x x}, \phi^{\prime}\right), \quad \forall \phi \in S_{h} .
$$

Note that for $1 \leq i \leq N$,

$$
\begin{aligned}
\left(\omega, \phi_{i}\right)= & \left(\rho_{t x}, \phi_{i}^{\prime}\right)+3\left(u \rho_{x}, \phi_{i}\right)+\left(u_{x} \rho_{x}, \phi_{i}^{\prime}\right)+\left(u \rho_{x x}, \phi_{i}^{\prime}\right) \\
= & \left(\left(Q_{h} u_{t}\right)_{x}-u_{t x}, \phi_{i}^{\prime}\right)+3\left(u\left(Q_{h} u\right)_{x}-u u_{x}, \phi_{i}\right) \\
& +\left(u_{x}\left(Q_{h} u\right)_{x}-u_{x}^{2}, \phi_{i}^{\prime}\right)+\left(\left(u\left(Q_{h} u\right)_{x x}-u u_{x x}, \phi_{i}^{\prime}\right) .\right.
\end{aligned}
$$

Therefore, from (2.4) it follows that

$$
\begin{aligned}
\left(\omega, \phi_{i}\right)= & -\left(Q_{h} u_{t x x}-u_{t x x}, \phi_{i}\right)+3\left(Q_{h}\left(u u_{x}\right)-u u_{x}, \phi_{i}\right) \\
& -\left(Q_{h}\left(u_{x}^{2}\right)_{x}-\left(u_{x}^{2}\right)_{x}, \phi_{i}\right)-\left(Q_{h}\left(u u_{x x}\right)_{x}-\left(u u_{x x}\right)_{x}, \phi_{i}\right)+\gamma_{i},
\end{aligned}
$$

where $\max _{1 \leq i \leq N}\left|\gamma_{i}\right| \leq C h^{2 r-1}$. Hence, from (2.2), (2.1) it follows that

$$
\|\omega\| \leq C h^{r} .
$$

Putting $\phi=\psi$ in (3.6) and taking into account (2.1), (2.5), and (2.6) we get

$$
\|\psi\|_{1}^{2} \leq C\left(h^{r}\|\psi\|+h^{2 r-3 / 2}\|\psi\|+h^{2 r-5 / 2}\|\psi\|_{1}+h^{2 r-2}\|\psi\|_{1}\right)+\|\omega\|\|\psi\|,
$$

which, by (3.7) and the fact that $r \geq 3$ yields (3.4).

We now estimate the $L^{2}$-error of the semidiscrete scheme (3.1)-(3.2). 
Proposition 3.2. If $u$ is the solution (assumed to be sufficiently smooth) of (C-H-per in $[0,1] \times[0, T]$, then for $h$ sufficiently small, the semidiscrete problem (3.1)-(3.2) has, for $r \geq 3$, a unique solution $u_{h}:[0, T] \rightarrow S_{h}$. Moreover, there exists a constant $C$ independent of $h$ such that

$$
\max _{0 \leq t \leq T}\left\|u(t)-u_{h}(t)\right\| \leq C h^{r} .
$$

Proof. It is clear that the ode system represented by (3.1)-(3.2) has a unique solution $u_{h}$ locally in $t$. While the solution exists, taking $\phi=u_{h}$ in (3.1) and using integration by parts gives

$$
\frac{1}{2} \frac{d}{d t}\left(\left\|u_{h}\right\|^{2}+\left\|u_{h x}\right\|^{2}\right)+\frac{1}{2}\left(u_{h x}^{2}, u_{h x}\right)+\left(u_{h} u_{h x x}, u_{h x}\right)=0 .
$$

Due to periodicity $\left(u_{h} u_{h x x}, u_{h x}\right)=\frac{1}{2}\left(u_{h}, \partial_{x}\left(u_{h x}^{2}\right)\right)=-\frac{1}{2}\left(u_{h x}, u_{h x}^{2}\right)$. Therefore, $\frac{d}{d t}\left\|u_{h}\right\|_{1}^{2}=0$, i.e. the $H^{1}$ norm of the semidiscrete solution is conserved. By standard ode theory this implies that $u_{h}$ exists in any finite temporal interval.

Let now $U=Q_{h} u$ and $\theta=U-u_{h}$. From (3.5), (3.1) we have

$$
A\left(\theta_{t}, \phi\right)+3\left(U U_{x}-u_{h} u_{h x}, \phi\right)+\frac{1}{2}\left(U_{x}^{2}-u_{h x}^{2}, \phi^{\prime}\right)+\left(U U_{x x}-u_{h} u_{h x x}, \phi^{\prime}\right)=A(\psi, \phi), \quad \forall \phi \in S_{h} .
$$

Since

$$
\begin{aligned}
& U U_{x}-u_{h} u_{h x}=U U_{x}-(U-\theta)\left(U_{x}-\theta_{x}\right)=(U \theta)_{x}-\theta \theta_{x}, \\
& U_{x}^{2}-u_{h x}^{2}=\theta_{x}\left(U_{x}+u_{h x}\right)=\theta_{x}\left(2 U_{x}-\theta_{x}\right)=2 U_{x} \theta_{x}-\theta_{x}^{2}, \\
& U U_{x x}-u_{h} u_{h x x}=U U_{x x}-(U-\theta)\left(U_{x x}-\theta_{x x}\right)=U \theta_{x x}+U_{x x} \theta-\theta \theta_{x x},
\end{aligned}
$$

(3.9) implies for $\phi=\theta$ that

$$
\frac{1}{2} \frac{d}{d t}\|\theta\|_{1}^{2}+3\left((U \theta)_{x}, \theta\right)+\left(U_{x} \theta_{x}, \theta_{x}\right)-\frac{1}{2}\left(\theta_{x}^{2}, \theta_{x}\right)+\left(U \theta_{x x}, \theta_{x}\right)+\left(U_{x x} \theta, \theta_{x}\right)-\left(\theta \theta_{x x}, \theta_{x}\right)=A(\psi, \theta) .
$$

Using again integration by parts we get

$$
\frac{1}{2} \frac{d}{d t}\|\theta\|_{1}^{2}+\frac{3}{2}\left(U_{x} \theta, \theta\right)+\frac{1}{2}\left(U_{x} \theta_{x}, \theta_{x}\right)+\left(U_{x x} \theta, \theta_{x}\right)=A(\psi, \theta) .
$$

Therefore, by (2.7) and (3.4) it follows that

$$
\frac{d}{d t}\|\theta\|_{1}^{2} \leq C\left(h^{2 r}+\|\theta\|_{1}^{2}\right), \quad t \in[0, T] .
$$

Since $\theta(0)=0$ by (3.2), Gronwall's lemma gives that $\|\theta\|_{1} \leq C h^{r}$ on $[0, T]$; hence (3.8) follows from (2.1).

Remark The error estimate (3.8) is still valid if $u_{h}(0)$ is taken as the $H^{1}$ ('elliptic') projection of $u_{0}$ on $S_{h}$, i.e. defined as $u_{h}(0)=v_{h}$, where

$$
A\left(v_{h}, \phi\right)=A\left(u_{0}, \phi\right), \quad \forall \phi \in S_{h} .
$$

Indeed, if $\varepsilon=Q_{h} u_{0}-v_{h}$ we have for $\phi \in S_{h}$

$$
A(\varepsilon, \phi)=A\left(Q_{h} u_{0}, \phi\right)-A\left(u_{0}, \phi\right)=\left(Q_{h} u_{0}-u_{0}, \phi\right)+(\gamma, \phi),
$$

where $\gamma \in S_{h}$ is defined for $\phi \in S_{h}$ by

$$
\left.(\gamma, \phi)=\left(\left(Q_{h} u_{0}\right)^{\prime}-u_{0}^{\prime}, \phi^{\prime}\right)\right)
$$

Therefore, by (2.4) for $1 \leq i \leq N$,

$$
\left(\gamma, \phi_{i}\right)=\left(\left(Q_{h} u_{0}\right)^{\prime}-u_{0}^{\prime}, \phi_{i}\right)=-\left(Q_{h} u_{0}^{\prime \prime}-u_{0}^{\prime \prime}, \phi_{i}\right)+\beta_{i},
$$

where $\max _{1 \leq i \leq N}\left|\beta_{i}\right|=O\left(h^{2 r-1}\right)$. Hence, by (2.1) and (2.2), $\|\gamma\| \leq C h^{r}$. It follows from (3.12) with $\phi=\varepsilon$ that $\|\varepsilon\|_{1}^{2} \leq C h^{r}\|\varepsilon\|$, i.e. that $\|\varepsilon\|_{1} \leq C h^{r}$. This implies for $\theta=U-u_{h}=Q_{h} u-u_{h}$, that $\|\theta(0)\|_{1}=\left\|Q_{h} u_{0}-v_{h}\right\|_{1} \leq C h^{r}$ and the application of Gronwall's lemma to (3.10) gives again $\|\theta\|_{1} \leq C h^{r}$ on $[0, T]$, i.e. that (3.8) still holds. Computing $v_{h}$ from (3.11) requires just the usual B-spline basis and not the special basis $\left\{\phi_{i}\right\}$. 


\section{A MOdified GALERKIN MEthod FOR THE PERIOdIC PROBlEM IN SYSTEM Form}

In this section we consider a modified Galerkin method for the periodic ivp for $\mathrm{CH}$, that works also if $r=2$, i.e. when the finite element space consists of 1-periodic, continuous, piecewise linear functions on a uniform mesh in $[0,1]$, consequently being a subspace of $H_{p e r}^{1}$.

For this purpose, following [13, we write (C-H-per) in system form for two 1-periodic functions $m$ and $u$ as follows:

(C-H-s-per)

$$
\begin{aligned}
& m=u-u_{x x}, \\
& m_{t}+(m u)_{x}+m u_{x}=0, \quad 0 \leq x \leq 1, \quad 0 \leq t \leq T, \\
& u(x, 0)=u_{0}(x), \quad m(x, 0)=u_{0}(x)-u_{0}^{\prime \prime}(x), \quad 0 \leq x \leq 1 .
\end{aligned}
$$

We will discretize C-H-s-per in space using approximations $u_{h}, m_{h}$ of $u, m$ that take values for $0 \leq t \leq T$ in $S_{h}$ for $r \geq 2$. This will yield a modified Galerkin method defined for $0 \leq t \leq T$ by

$$
\begin{aligned}
& \left(m_{h}, \phi\right)=\left(u_{h}, \phi\right)+\left(u_{h x}, \phi^{\prime}\right), \quad \forall \phi \in S_{h}, \\
& \left(m_{h t}, \phi\right)+\left(\left(m_{h} u_{h}\right)_{x}, \phi\right)+\left(m_{h} u_{h x}, \phi\right)=0, \quad \forall \phi \in S_{h},
\end{aligned}
$$

with initial value

$$
\left(m_{h}(0), \phi\right)=\left(u_{0}, \phi\right)+\left(u_{0}^{\prime}, \phi^{\prime}\right), \quad \phi \in S_{h}
$$

i.e. with $m_{h}(x, 0)$ taken as the $L^{2}$-projection of $m(x, 0)$ on $S_{h}$. Compatibility with (4.1) and (4.3) imply that $u_{h}(0)$ satisfies for $\phi \in S_{h}$

$$
A\left(u_{h}(0), \phi\right)=A\left(u_{0}, \phi\right)
$$

i.e. that $u_{h}(0)$ is the $H^{1}$ projection of $u_{0}$ in $S_{h}$. We first establish the consistency of (4.1)-(4.3) with C-H-s-per.

Lemma 4.1. Let $(m, u)$, the solution of C-H-s-per in $[0,1] \times[0, T]$, be sufficiently smooth and suppose that $r \geq 2$. If $M=Q_{h} m, U=Q_{h} u$ and $\psi, \zeta:[0 . T] \rightarrow S_{h}$ are such that

$$
\begin{aligned}
& (U, \phi)+\left(U_{x}, \phi^{\prime}\right)-(M, \phi)=(\psi, \phi), \quad \forall \phi \in S_{h}, \\
& \left(M_{t}, \phi\right)+\left((M U)_{x}, \phi\right)+\left(M U_{x}, \phi\right)=(\zeta, \phi) \quad \forall \phi \in S_{h},
\end{aligned}
$$

then, there exists a constant $C$ independent of $h$, such that

$$
\max _{0 \leq t \leq T}(\|\psi(t)\|+\|\zeta(t)\|) \leq C h^{r} .
$$

Proof. Let $\rho=Q_{h} u-u=U-u$, and $\sigma=Q_{h} m-m=M-m$. Then

$$
\begin{aligned}
& (\psi, \phi)=(\rho, \phi)+\left(\rho_{x}, \phi^{\prime}\right)-(\sigma, \phi)=(\rho-\sigma, \phi)+(\widetilde{\psi}, \phi), \quad \forall \phi \in S_{h}, \\
& (\zeta, \phi)=\left(\sigma_{t}, \phi\right)+\left((M U)_{x}-(m u)_{x}, \phi\right)+\left(M U_{x}-m u_{x}, \phi\right), \quad \forall \phi \in S_{h}
\end{aligned}
$$

where $\widetilde{\psi}:[0, T] \rightarrow S_{h}$ satisfies

$$
(\widetilde{\psi}, \phi)=\left(\rho_{x}, \phi^{\prime}\right), \quad \forall \phi \in S_{h}
$$

Since

$$
\begin{aligned}
& M U-m u=(m+\sigma)(u+\rho)-m u=m \rho+u \sigma+\sigma \rho, \\
& M U_{x}-m u_{x}=(m+\sigma)\left(u_{x}+\rho_{x}\right)-m u_{x}=m \rho_{x}+u_{x} \sigma+\sigma \rho_{x},
\end{aligned}
$$

it follows from the second equation of (4.7) that

$$
(\zeta, \phi)=\left(\sigma_{t}, \phi\right)+\left(m_{x} \rho, \phi\right)+2\left(u_{x} \sigma, \phi\right)+\left(\sigma_{x} \rho, \phi\right)+2\left(\sigma \rho_{x}, \phi\right)+(\widetilde{\zeta}, \phi), \quad \forall \phi \in S_{h},
$$

where $\widetilde{\zeta}:[0, T] \rightarrow S_{h}$ is defined as

$$
(\widetilde{\zeta}, \phi)=2\left(m \rho_{x}, \phi\right)+\left(u \sigma_{x}, \phi\right), \quad \forall \phi \in S_{h} .
$$

It follows from (4.8) for $1 \leq i \leq N$,

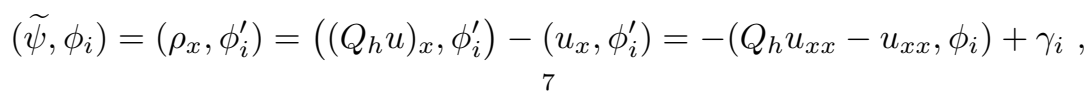


where, from (2.4), $\max _{1 \leq i \leq N}\left|\gamma_{i}\right| \leq C h^{2 r-1}$. Therefore, from (2.2) and (2.1) we see that

$$
\|\widetilde{\psi}\| \leq C h^{r} \text {. }
$$

Putting $\phi=\psi$ in the first equation of (4.7) and taking into account (2.1) and (4.11) we get

$$
\|\psi\| \leq C h^{r} \text {. }
$$

In addition, from (4.10) it follows for $1 \leq i \leq N$

$$
\begin{aligned}
\left(\widetilde{\zeta}, \phi_{i}\right) & =2\left(m\left(Q_{h} u\right)_{x}, \phi_{i}\right)-2\left(m u_{x}, \phi_{i}\right)+\left(u\left(Q_{h} m\right)_{x}, \phi_{i}\right)-\left(u m_{x}, \phi_{i}\right) \\
& =2\left(Q_{h}\left(m u_{x}\right)-m u_{x}, \phi_{i}\right)+\left(Q_{h}\left(u m_{x}\right)-u m_{x}, \phi_{i}\right)+\widetilde{\gamma}_{i},
\end{aligned}
$$

where, by (2.4), $\max _{1 \leq i \leq N}\left|\widetilde{\gamma}_{i}\right| \leq C h^{2 r+1}$. Consequently, from (2.2) and (2.1) we have

$$
\|\widetilde{\zeta}\| \leq C h^{r} .
$$

Finally, if we take $\phi=\zeta$ in (4.9), we obtain, in view of (4.13),

$$
\|\zeta\| \leq C h^{r}
$$

which, together with (4.12), establishes (4.6).

We proceed now to derive an $L^{2}$-error estimate for the solution of the semidiscrete problem (4.1)-(4.3).

Proposition 4.2. If the solution $(m, u)$ of (C-H-s-per) on $[0,1] \times[0, T]$ is sufficiently smooth, then, for $h$ sufficiently small, the semidiscrete problem (4.1)-(4.3) has, for $r \geq 2$, a unique solution on $[0, T]$ that satisfies

$$
\max _{0 \leq t \leq T}\left(\left\|m(t)-m_{h}(t)\right\|+\left\|u(t)-u_{h}(t)\right\|\right) \leq C h^{r} .
$$

Proof. For every $t \geq 0$ we may solve the linear discrete problem (4.1) and express $u_{h}$ in terms of $m_{h}$. Upon substituting $u_{h}$ in (4.2), we easily see that the resulting initial-value problem (4.2)-(4.3) has a unique solution $m_{h}$ locally in $t$. While this solution exists, putting $M=Q_{h} m, U=Q_{h} u, \theta=U-u_{h}$, and $\xi=M-m_{h}$, from (4.5), (4.1), (4.2) we obtain

$$
\begin{aligned}
& (\theta, \phi)+\left(\theta_{x}, \phi^{\prime}\right)-(\xi, \phi)=(\psi, \phi), \quad \forall \phi \in S_{h}, \\
& \left(\xi_{t}, \phi\right)+\left(\left(M U-m_{h} u_{h}\right)_{x}, \phi\right)+\left(M U_{x}-m_{h} u_{h x}, \phi\right)=(\zeta, \phi), \quad \forall \phi \in S_{h} .
\end{aligned}
$$

With $\phi=\theta$ in the first equation above we see, in view of (4.6), that

$$
\|\theta\|_{1} \leq\|\xi\|+C h^{r} .
$$

Now

$$
\begin{aligned}
& M U-m_{h} u_{h}=M U-(M-\xi)(U-\theta)=M \theta+U \xi-\theta \xi, \\
& M U_{x}-m_{h} u_{h x}=M U_{x}-(M-\xi)\left(U_{x}-\theta_{x}\right)=M \theta_{x}+U_{x} \xi-\theta_{x} \xi .
\end{aligned}
$$

Hence, from the second equation in (4.15), putting $\phi=\xi$ we obtain

$$
\frac{1}{2} \frac{d}{d t}\|\xi\|^{2}+\left((M \theta)_{x}, \xi\right)+\left((U \xi)_{x}, \xi\right)-\left((\theta \xi)_{x}, \xi\right)+\left(M \theta_{x}, \xi\right)+\left(U_{x} \xi, \xi\right)-\left(\theta_{x} \xi, \xi\right)=(\zeta, \xi) .
$$

Using integration by parts we see that $\left((U \xi)_{x}, \xi\right)=-\left(U \xi, \xi_{x}\right)=\frac{1}{2}\left(U_{x} \xi, \xi\right)$, and similarly, $\left((\theta \xi)_{x}, \xi\right)=\frac{1}{2}\left(\theta_{x} \xi, \xi\right)$. Therefore, we may write the above equation as

$$
\frac{1}{2} \frac{d}{d t}\|\xi\|^{2}+\left(M_{x} \theta, \xi\right)+2\left(M \theta_{x}, \xi\right)+\frac{3}{2}\left(U_{x} \xi, \xi\right)-\frac{3}{2}\left(\theta_{x} \xi, \xi\right)=(\zeta, \xi) .
$$

Consequently, from (2.7), (4.6), (4.16), we get for some constant $C$ independent of $h$, that

$$
\frac{d}{d t}\|\xi\|^{2} \leq C\left(\|\xi\|^{2}+h^{r}\|\xi\|\right)+3\left|\left(\theta_{x} \xi, \xi\right)\right| .
$$

The definition of $m_{h}(0)$ by (4.3) implies that for $\xi(0)=Q_{h} m(0)-m_{h}(0)$ we have if $1 \leq i \leq N$

$$
\begin{aligned}
\left(\xi(0), \phi_{i}\right) & =\left(Q_{h} m(0)-m_{h}(0), \phi_{i}\right)=\left(Q_{h}\left(u_{0}-u_{0}^{\prime \prime}\right), \phi_{i}\right)-\left(u_{0}, \phi_{i}\right)-\left(u_{0}^{\prime}, \phi_{i}^{\prime}\right) \\
& =\left(Q_{h} u_{0}-u_{0}, \phi_{i}\right)-\left(Q_{h} u_{0}^{\prime \prime}-u_{0}^{\prime \prime}, \phi_{i}\right) .
\end{aligned}
$$

Therefore, by (2.2) and (2.1) we conclude that

$$
\|\xi(0)\| \underset{8}{\leq} C h^{r}
$$


from which, by inverse properties, we see that $\|\xi(0)\|_{\infty}=O\left(h^{r-1 / 2}\right)$. By continuity we may infer that there is a maximal time $t_{h} \in(0, T]$ such that the solution of (4.1)-(4.3) exists for $0 \leq t \leq t_{h}$ and satisfies

$$
\max _{0 \leq t \leq t_{h}}\|\xi(t)\|_{\infty} \leq 1 \text {. }
$$

Therefore, from (4.17) it follows that

$$
\frac{d}{d t}\|\xi\|^{2} \leq C\left(\|\xi\|^{2}+h^{r}\|\xi\|\right)+3\|\theta\|_{1}\|\xi\| \leq C\left(h^{2 r}+\|\xi\|^{2}\right),
$$

for $t \leq t_{h}$. From this inequality and Gronwall's lemma we infer that

$$
\|\xi(t)\| \leq C_{T}\left(\|\xi(0)\|+h^{r}\right),
$$

for $t \leq t_{h}$. Hence, by (4.18)

$$
\|\xi(t)\| \leq C_{T} h^{r}, \quad 0 \leq t \leq t_{h},
$$

from which, by inverse properties we conclude that

$$
\|\xi(t)\|_{\infty} \leq C_{T} h^{r-1 / 2},
$$

for $t \leq t_{h}$. Therefore, if $h$ is taken sufficiently small, $t_{h}$ is not maximal and one can consequently take $t_{h}=T$. Hence, (4.20) holds for $t \in[0, T]$. By (4.16), $\|\theta\|_{1} \leq C h^{r}$ on $[0, T]$ and (4.14) follows in view of (2.1).

\section{A Galerkin method For the initial-Boundary-VAlue PROBlem}

In this section we consider the following initial-boundary value problem (ibvp) for the Camassa-Holm equation. For $0 \leq t \leq T$ and $0 \leq x \leq 1$ we seek $u=u(x, t)$ satisfying

$$
\begin{aligned}
u_{t}-u_{t x x}+3 u u_{x} & =2 u_{x} u_{x x}+u u_{x x x}, \quad 0 \leq x \leq 1, \quad 0 \leq t \leq T, \\
u(x, 0) & =u_{0}(x), \quad 0 \leq x \leq 1, \\
u(0, t)=u_{x x}(0, t) & =u(1, t)=u_{x x}(1, t)=0, \quad 0 \leq t \leq T .
\end{aligned}
$$

This ibvp was first analyzed by Kwek et al. [33, who showed that there exists $T>0$ such that it has a unique solution which is a continuous map of $[0, T]$ into $\mathcal{B}$, where $\mathcal{B}=\left\{v \in H^{4}(0,1): v(0)=v^{\prime \prime}(0)=v(1)=\right.$ $\left.v^{\prime \prime}(1)=0\right\}$. The form of the boundary conditions suggests that the problem should be naturally studied in its $m-u$ system form, and this was actually done in 33 . Consequently, we seek $u=u(x, t)$ and $m=m(x, t)$, defined for $(x, t) \in[0,1] \times[0, T]$ and satisfying

$$
\begin{aligned}
& m=u-u_{x x}, \\
& m_{t}+u m_{x}+2 u_{x} m=0, \quad 0 \leq x \leq 1, \quad 0 \leq t \leq T, \\
& m(0, t)=m(1, t)=u(0, t)=u(1, t)=0,0 \leq t \leq T, \\
& u(x, 0)=u_{0}(x), \quad m(x, 0)=m_{0}(x), 0 \leq x \leq 1,
\end{aligned}
$$

where $m_{0}=u_{0}-u_{0}^{\prime \prime}$.

In the sequel we will analyze a Galerkin-finite element method for the ibvp (C-H-s-b) and also make some remarks on the numerical solution of the ibvp ( $\mathrm{C}-\mathrm{H}-\mathrm{b})$ for the single equation by a standard Galerkin method. We assume that these ibvp's possess solutions that are smooth enough for the purposes of the error estimation.

Letting $A(\cdot, \cdot)$ be the bilinear form considered in Section 3, but now defined for $v, w \in \stackrel{H}{H}^{1}$ by

$$
A(v, w)=(v, w)+\left(v^{\prime}, w^{\prime}\right),
$$

i.e. as the $H^{1}$ inner product, we may write (C-H-s-b) in weak form for $0 \leq t \leq T$ as

$$
(m, v)=A(u, v), \quad \forall v \in \stackrel{\circ}{H}^{1},
$$

and

$$
\left(m_{t}, v\right)=-\left(u m_{x}, v\right)-\left(2 u_{x} m, v\right), \quad \forall v \in \stackrel{\circ}{H}^{1} .
$$

In order to define the numerical method, let $0=x_{1}<x_{2}<\cdots<x_{N+1}=1$ be a quasiuniform partition of $[0,1]$ with maximum meshlength $h$. For integers $r \geq 2$ and $0 \leq \mu \leq r-2$ let $S_{h}^{r, \mu}=\left\{\phi \in C^{\mu}[0,1]\right.$ : $\left.\left.\phi\right|_{\left[x_{i}, x_{i+1}\right]} \in \mathbb{P}_{r-1}, i=1,2, \ldots, N\right\}$ and $S_{h}=S_{h}^{r, \mu} \cap \stackrel{\circ}{H}^{1}$. By $R_{h}: \stackrel{\circ}{H}^{1} \rightarrow S_{h}$ denote the elliptic projection 
onto $S_{h}$ defined as usual for $v \in \stackrel{\circ}{H}^{1}$ as $A\left(R_{h} v, \phi\right)=A(v, \phi)$ for all $\phi \in S_{h}$ and let $P_{h}: L^{2} \rightarrow S_{h}$ be the $L^{2}$ projection onto $S_{h}$. From well-known approximation properties of the finite element spaces $S_{h}$, 21], [43], it follows that

$$
\begin{gathered}
\left\|R_{h} v-v\right\|_{j} \leq C h^{k-j}\|v\|_{k}, \quad j=0,1, \quad 2 \leq k \leq r, \quad v \in H^{k} \cap H_{0}^{1}, \\
\left\|P_{h} v-v\right\|_{j} \leq C h^{k-j}\|v\|_{k}, \quad j=0,1, \quad 2 \leq k \leq r, \quad v \in H^{k} \cap H_{0}^{1} .
\end{gathered}
$$

We may also asume the following stability properties of $R_{h}$ and $P_{h}$, valid for functions in the spaces implied by the indicated norms, that also vanish at $x=0$ and $x=1$.

$$
\begin{aligned}
\left\|R_{h} v\right\|_{\infty} & \leq C\|v\|_{\infty}, \quad\left\|R_{h} v\right\|_{1, \infty} \leq C\|v\|_{1, \infty}, \\
\left\|P_{h} v\right\|_{1} & \leq C\|v\|_{1}, \quad\left\|P_{h} v\right\|_{\infty} \leq C\|v\|_{\infty} .
\end{aligned}
$$

(For the stability of $R_{h}$ in the $\|\cdot\|_{\infty}$ norm and of $P_{h}$ in $\stackrel{\circ}{H}^{1}$ cf. 44. For the stability of $P_{h}$ in $L^{\infty}$ cf. [22, while for that of $R_{h}$ in the $\|\cdot\|_{1, \infty}$ norm, cf. [42, 11.) In addition, note that the inverse inequalities $\|\chi\|_{1} \leq C h^{-1}\|\chi\|,\|\chi\|_{j, \infty} \leq C h^{-(j+1 / 2)}\|\chi\|, j=0,1$, hold for $\chi \in S_{h}$. The semidiscrete approximation of (C)-H-s-b) is now defined as follows. We seek $m_{h}, u_{h}:[0, T] \rightarrow S_{h}$ satisfying for $0 \leq t \leq T$

$$
\begin{aligned}
& \left(m_{h}, \phi\right)=A\left(u_{h}, \phi\right), \quad \forall \phi \in S_{h}, \\
& \left(m_{h t}, \chi\right)=-\left(u_{h} m_{h x}, \chi\right)-2\left(u_{h x} m_{h}, \chi\right), \quad \forall \chi \in S_{h},
\end{aligned}
$$

with initial conditions

$$
m_{h}(0)=P_{h}\left(u_{0}-u_{0}^{\prime \prime}\right), \quad u_{h}(0)=R_{h} u_{0} .
$$

Proposition 5.1. Let $r \geq 2$ and suppose that the ibvp (C-H-s-b) has a unique solution $(m, u)$, with $m \in$ $C\left(0, T ; C^{r-2} \cap \stackrel{\circ}{H}^{1}\right), u \in C\left(0, T ; C^{r} \cap \stackrel{\circ}{H}^{1}\right)$. Then, if $h$ is sufficiently small, the semidiscrete problem (5.8) (5.10) has a unique solution $\left(m_{h}, u_{h}\right)$ in $[0, T]$ for which

$$
\max _{0 \leq t \leq T}\left(\left\|m(t)-m_{h}(t)\right\|+\left\|u(t)-u_{h}(t)\right\|_{1}\right) \leq C h^{r-1} .
$$

Proof. Let $\rho=u-R_{h} u, \theta=R_{h} u-u_{h}, \sigma=m-P_{h} m$, and $\xi=P_{h} m-m_{h}$. Then $u-u_{h}=\rho+\theta$, $m-m_{h}=\sigma+\xi$, and it suffices to show that for $0 \leq t \leq T$

$$
\|\xi\|+\|\theta\|_{1} \leq C h^{r-1} .
$$

It is clear that the initial value o.d.e. problem represented by (5.8)-(5.10) has a unique solution $\left(m_{h}, u_{h}\right)$ locally in $t$. While this solution exists, subtracting (5.8) from (5.2) (with $v=\phi$ ) and (5.9) from (5.3) (with $w=\chi)$ gives

$$
\begin{aligned}
& (\xi, \phi)=A(\theta, \phi), \quad \forall \phi \in S_{h}, \\
& \left(\xi_{t}, \chi\right)=-\left(u m_{x}-u_{h} m_{h x}, \chi\right)-2\left(u_{x} m-u_{h x} m_{h}, \chi\right), \quad \forall \chi \in S_{h} .
\end{aligned}
$$

Since

$$
\begin{aligned}
& u m_{x}-u_{h} m_{h x}=\left(m_{x}-m_{h x}\right) u+\left(u-u_{h}\right) m_{x}-\left(u-u_{h}\right)\left(m_{x}-m_{h x}\right), \\
& u_{x} m-u_{h x} m_{h}=\left(m-m_{h}\right) u_{x}+\left(u_{x}-u_{h x}\right) m-\left(u_{x}-u_{h x}\right)\left(m-m_{h}\right),
\end{aligned}
$$

it follows that

$$
\begin{aligned}
& u m_{x}-u_{h} m_{h x}=\sigma_{x} u+\xi_{x} u+\rho m_{x}+\theta m_{x}-\rho \sigma_{x}-\rho \xi_{x}-\theta \sigma_{x}-\theta \xi_{x}, \\
& u_{x} m-u_{h x} m_{h}=\sigma u_{x}+\xi u_{x}+\rho_{x} m+\theta_{x} m-\rho_{x} \sigma-\rho_{x} \xi-\theta_{x} \sigma-\theta_{x} \xi
\end{aligned}
$$

and therefore

$$
\begin{aligned}
\left(\xi_{t}, \chi\right)= & -\left(\sigma_{x} u+2 \sigma u_{x}, \chi\right)-\left(\xi_{x} u+2 \xi u_{x}, \chi\right)-\left(\rho m_{x}+2 \rho_{x} m, \chi\right)-\left(\theta m_{x}+2 \theta_{x} m, \chi\right) \\
& +\left(\rho \sigma_{x}+2 \rho_{x} \sigma, \chi\right)+\left(\rho \xi_{x}+2 \rho_{x} \xi, \chi\right)+\left(\theta \sigma_{x}+2 \theta_{x} \sigma, \chi\right)+\left(\theta \xi_{x}+2 \theta_{x} \xi, \chi\right), \forall \chi \in S_{h} .
\end{aligned}
$$

Putting $\chi=\xi$ in this relation we get

$$
\left(\xi_{t}, \xi\right)=-w_{1}-w_{2}-w_{3}-w_{4}+w_{5}+w_{6}+w_{7}+w_{8},
$$


where

$$
\begin{aligned}
& w_{1}=\left(\sigma_{x} u+2 \sigma u_{x}, \xi\right), w_{2}=\frac{3}{2}\left(\xi u_{x}, \xi\right), w_{3}=\left(\rho m_{x}+2 \rho_{x} m, \xi\right), w_{4}=\left(\theta m_{x}+2 \theta_{x} m, \xi\right), \\
& w_{5}=\left(\rho \sigma_{x}+2 \rho_{x} \sigma, \xi\right) w_{6}=\frac{3}{2}\left(\rho_{x} \xi, \xi\right), w_{7}=\left(\theta \sigma_{x}+2 \theta_{x} \sigma, \xi\right), w_{8}=\frac{3}{2}\left(\theta_{x} \xi, \xi\right) .
\end{aligned}
$$

Taking into account (5.4) and (5.5) we may estimate $w_{1}, w_{2}, w_{3}$ as follows:

$$
\begin{aligned}
& \left|w_{1}\right| \leq\left(\left\|\sigma_{x}\right\|\|u\|_{\infty}+2\|\sigma\|\left\|u_{x}\right\|_{\infty}\right)\|\xi\| \leq C h^{r-1}\|\xi\|, \\
& \left|w_{2}\right| \leq \frac{3}{2}\left\|u_{x}\right\|_{\infty}\|\xi\|^{2} \leq C\|\xi\|^{2}, \\
& \left|w_{3}\right| \leq\left(\|\rho\|\left\|m_{x}\right\|_{\infty}+2\left\|\rho_{x}\right\|\|m\|_{\infty}\right)\|\xi\| \leq C h^{r-1}\|\xi\| .
\end{aligned}
$$

To estimate $w_{4}$ we take into account (5.12), which implies that

$$
\|\theta\|_{1} \leq\|\xi\| \text {. }
$$

Hence

$$
\left|w_{4}\right| \leq\left(\left\|m_{x}\right\|_{\infty}\|\theta\|+2\|m\|_{\infty}\left\|\theta_{x}\right\|\right)\|\xi\| \leq C\|\xi\|^{2} .
$$

For $w_{5}$ and $w_{6}$, using the stability of $R_{h}$ in $L^{\infty}$ and $W_{\infty}^{1}$, cf. (5.6), and (5.5) gives

$$
\begin{aligned}
& \left|w_{5}\right| \leq\left(\|\rho\|_{\infty}\left\|\sigma_{x}\right\|+2\left\|\rho_{x}\right\|_{\infty}\|\sigma\|\right)\|\xi\| \leq C h^{r-1}\|\xi\|, \\
& \left|w_{6}\right| \leq \frac{3}{2}\left\|\rho_{x}\right\|_{\infty}\|\xi\|^{2} \leq C\|\xi\|^{2} .
\end{aligned}
$$

To estimate $w_{7}$, by Sobolev's inequality, (5.18), and the stability of $P_{h}$ in $\stackrel{\circ}{H}^{1}$ and $L^{\infty}$, cf. (5.7), we get

$$
\left|w_{7}\right| \leq\left(\|\theta\|_{\infty}\left\|\sigma_{x}\right\|+2\left\|\theta_{x}\right\|\|\sigma\|_{\infty}\right)\|\xi\| \leq C\|\xi\|^{2} .
$$

In order to estimate $w_{8}$, we let $0<t_{h}<T$ be the maximal time such that the solution of (5.8)-(5.10) exists and satisfies $\max _{0 \leq t \leq t_{h}}\|\xi(t)\|_{\infty} \leq 1$. Then, by (5.18)

$$
\left|w_{8}\right| \leq \frac{3}{2}\left\|\theta_{x}\right\|\|\xi\|_{\infty}\|\xi\| \leq C\|\xi\|^{2} .
$$

In view of (5.17)-(5.23), (5.16) gives for $0 \leq t \leq t_{h}$

$$
\frac{d}{d t}\|\xi(t)\|^{2} \leq C\left(h^{2 r-2}+\|\xi(t)\|^{2}\right) .
$$

Hence Gronwall's lemma implies, for some constant $C=C(T)$, that

$$
\|\xi(t)\| \leq C h^{r-1}, \quad 0 \leq t \leq t_{h} .
$$

Since $\|\xi(t)\|_{\infty} \leq C h^{-1 / 2}\|\xi\| \leq C h^{r-3 / 2}$ from (15.24) , it follows that for $h$ small enough $\|\xi\|_{\infty}<1$ that implies $t_{h}$ was not maximal and that the argument may be repeated up to $t=T$. It follows that (5.24) holds on $[0, T]$, and by (5.18), we get that $\|\xi\|+\|\theta\|_{1}=O\left(h^{r-1}\right)$ on $[0, T]$, proving (5.11).

The rate of convergence $r-1$ in Proposition 5.1 is optimal for the error $u-u_{h}$ in the $H^{1}$ norm. In the $L^{2}$ norm we prove in the following proposition, using a duality argument, a suboptimal convergence rate estimate.

Proposition 5.2. Under the hypotheses of Proposition 5.1 there holds

$$
\max _{0 \leq t \leq T}\left\|u(t)-u_{h}(t)\right\| \leq C h^{r-1 / 2} .
$$

Proof. We use the notation of the proof of Proposition 5.1] If $v$ is the solution of the problem

$$
\begin{gathered}
v-v_{x x}=0, \\
v(0, t)=v(1, t)=0,
\end{gathered}
$$

then, taking into account (5.12) and (5.13), it follows that

$$
\left(\theta_{t}, \theta\right)=A\left(\theta_{t}, v\right)=A\left(\theta_{t}, R_{h} v\right)=\left(\xi_{t}, R_{h} v\right)=-\left(e, R_{h} v-v\right)-(e, v),
$$

where $e=e_{1}+2 e_{2}$, and

$$
\begin{aligned}
& e_{1}=\left(m_{x}-m_{h x}\right) u+\left(u-u_{h}\right) m_{x}-\left(u-u_{h}\right)\left(m_{x}-m_{h x}\right), \\
& e_{2}=\left(m-m_{h}\right) u_{x}+\left(u_{x}-u_{h x}\right) m-\left(u_{x}-u_{h x}\right)\left(m-m_{h}\right) .
\end{aligned}
$$


(The last inequality of (5.27) follows from noting that $e=e_{1}+2 e_{2}=u m_{x}-u_{h} m_{h x}+2 m u_{x}-2 m_{h} u_{h x}$, and (5.13) with $\chi=R_{h} v$.) We now estimate the last two terms in (5.27). In view of (5.4) and (5.26) we have

$$
\left|\left(e, R_{h} v-v\right)\right| \leq\|e\|\left\|R_{h} v-v\right\| \leq C h^{2}\|v\|_{2}\|e\| \leq C h^{2}\|\theta\|\|e\| .
$$

Using (5.11), Sobolev's inequality, and e.g. (5.5) and the inverse inequalities in $S_{h}$ we see that

$$
\begin{aligned}
\left\|e_{1}\right\| & \leq\|u\|_{\infty}\left\|m_{x}-m_{h x}\right\|+\left\|m_{x}\right\|_{\infty}\left\|u-u_{h}\right\|+\left\|u-u_{h}\right\|_{\infty}\left\|m_{x}-m_{h x}\right\| \\
& \leq C\left(h^{r-2}+h^{r-1}+h^{2 r-3}\right) \leq C h^{r-2} .
\end{aligned}
$$

Similarly $\left\|e_{2}\right\| \leq C h^{r-2}$, and (5.28) gives

$$
\left|\left(e, R_{h} v-v\right)\right| \leq C h^{r}\|\theta\| .
$$

The definition of $e$ gives

$$
\begin{aligned}
(e, v)= & \left(\sigma_{x} u+2 \sigma u_{x}, v\right)+\left(\rho m_{x}+2 \rho_{x} m, v\right)+\left(\theta m_{x}+2 \theta_{x} m, v\right)-\left(\rho \sigma_{x}+2 \rho_{x} \sigma, v\right) \\
& -\left(\rho \xi_{x}+2 \rho_{x} \xi, v\right)-\left(\theta \sigma_{x}+2 \theta_{x} \sigma, v\right)-\left(\theta \xi_{x}+2 \theta_{x} \xi, v\right)+\left(\xi_{x} u+2 \xi u_{x}, v\right) .
\end{aligned}
$$

Hence, integrating by parts,

$$
\begin{aligned}
(e, v)= & \left(\sigma, u_{x} v-u v_{x}\right)-\left(\rho, m_{x} v+2 m v_{x}\right)-\left(\theta, m_{x} v+2 m v_{x}\right)-\left(\rho \sigma_{x}+2 \rho_{x} \sigma, v\right) \\
& +\left(\xi, \rho v_{x}-\rho_{x} v\right)+\left(\sigma, \theta v_{x}-\theta_{x} v\right)+\left(\xi, \theta v_{x}-\theta_{x} v\right)+\left(\xi, u_{x} v-u v_{x}\right) \\
= & : \widetilde{w}_{1}+\widetilde{w}_{2}
\end{aligned}
$$

where

$$
\begin{aligned}
\widetilde{w}_{1}= & \left(\sigma, u_{x} v-u v_{x}\right)-\left(\rho, m_{x} v+2 m v_{x}\right)-\left(\theta, m_{x} v+2 m v_{x}\right)-\left(\rho \sigma_{x}+2 \rho_{x} \sigma, v\right) \\
& +\left(\xi, \rho v_{x}-\rho_{x} v\right)+\left(\sigma, \theta v_{x}-\theta_{x} v\right)+\left(\xi, \theta v_{x}-\theta_{x} v\right)
\end{aligned}
$$

and

$$
\widetilde{w}_{2}=\left(\xi, u_{x} v-u v_{x}\right)
$$

To estimate $\widetilde{w}_{1}$ note that

$$
\begin{aligned}
\left|\widetilde{w}_{1}\right| \leq & \|\sigma\|\left(\left\|u_{x}\right\|_{\infty}\|v\|+\|u\|_{\infty}\left\|v_{x}\right\|\right)+\|\rho\|\left(\left\|m_{x}\right\|_{\infty}\|v\|+2\|m\|_{\infty}\left\|v_{x}\right\|\right) \\
& +\|\theta\|\left(\left\|m_{x}\right\|_{\infty}\|v\|+2\|m\|_{\infty}\left\|v_{x}\right\|\right)+\left(\|\rho\|\left\|\sigma_{x}\right\|+2\left\|\rho_{x}\right\|\|\sigma\|\right)\|v\|_{\infty} \\
& +\|\xi\|\left(\|\rho\|\left\|v_{x}\right\|_{\infty}+\left\|\rho_{x}\right\|\|v\|_{\infty}\right)+\|\sigma\|\left(\|\theta\|\left\|v_{x}\right\|_{\infty}+\left\|\theta_{x}\right\|\|v\|_{\infty}\right) \\
& +\|\xi\|\left(\|\theta\|\left\|v_{x}\right\|_{\infty}+\left\|\theta_{x}\right\|\|v\|_{\infty}\right) .
\end{aligned}
$$

Therefore, taking into account (5.26), (5.5), (5.4), Sobolev's inequality, (5.11), and the inverse inequalities we see that

$$
\left|\widetilde{w}_{1}\right| \leq C\left(h^{2 r}+\|\theta\|^{2}\right) .
$$

In order to estimate $\widetilde{w}_{2}$, letting $f=u_{x} v-u v_{x}$ and recalling (5.12) we have

$$
\widetilde{w}_{2}=\left(\xi, u_{x} v-u v_{x}\right)=\left(\xi, P_{h} f\right)=A\left(\theta, P_{h} f-f\right)+\left(\theta, f-f_{x x}\right) .
$$

Since $f_{x x}=\left(u_{x x} v-u v_{x x}\right)_{x}=(-m v+u \theta)_{x}$, integration by parts gives

$$
\widetilde{w}_{2}=A\left(\theta, P_{h} f-f\right)+(\theta, f)+\left(\theta,(m v)_{x}\right)+\frac{1}{2}\left(u_{x} \theta, \theta\right) .
$$

Hence,

$$
\left|\widetilde{w}_{2}\right| \leq\|\theta\|_{1}\left\|P_{h} f-f\right\|_{1}+\|\theta\|\|f\|+\|\theta\|\left(\left\|m_{x}\right\|_{\infty}\|v\|+\|m\|_{\infty}\left\|v_{x}\right\|\right)+C\|\theta\|^{2} .
$$

Noting by (5.5) that $\left\|P_{h} f-f\right\|_{1} \leq C h\|f\|_{2}$ and taking into account (5.11) and (5.26) we see that

$$
\left|\widetilde{w}_{2}\right| \leq C\left(h^{2 r-1}+\|\theta\|^{2}\right) .
$$

Therefore, using this and (5.31), (5.27), and (5.29) give

$$
\frac{d}{d t}\|\theta\|^{2} \leq C\left(h^{2 r-1}+\|\theta\|^{2}\right) .
$$

Gronwall's lemma yields now that $\|\theta\| \leq C h^{r-1 / 2}$, proving (5.25). 
Remark It is also possible to construct a standard Galerkin method directly for the ibvp (C-H-b). For this purpose, using the notation introduced in the beginning of this section we will, as an example, discretize the problem in the space of cubic splines. i.e. in $S_{h}=\left\{\phi \in S_{h}^{4,2}: \phi(0)=\phi^{\prime \prime}(0)=\phi(1)=\phi^{\prime \prime}(1)=0\right\}$. We may assume now, cf. [21], 43], 1], that

$$
\left\|R_{h} v-v\right\|_{j} \leq C h^{4-j}\|v\|_{4}, \quad j=0,1,2,
$$

for $v \in H^{4}(0,1)$ satisfying $v(0)=v^{\prime \prime}(0)=v(1)=v^{\prime \prime}(1)=0$, and that $\|\chi\|_{k} \leq C h^{-1}\|\chi\|_{k-1}, k=1,2, \chi \in S_{h}$. The standard Galerkin semidiscrete approximation of ([C-H-b) in $S_{h}$ is then the map $u_{h}:[0, T] \rightarrow S_{h}$ defined by

$$
A\left(u_{h t}, \phi\right)=\left(u_{h x} u_{h x x}, \phi\right)-3\left(u_{h} u_{h x}, \phi\right)-\left(u_{h} u_{h x x}, \phi^{\prime}\right), \quad t \in[0, T], \quad \phi \in S_{h}
$$

with

$$
u_{h}(0)=R_{h} u_{0}
$$

Using standard techniques of error estimation we were able to prove only suboptimal error estimates for $u_{h}$. Specifically it holds that if $u$ is a classical solution of (C-H-b), then $u_{h}$ exists for $t \in[0, T]$ and satisfies

$$
\begin{aligned}
& \max _{0 \leq t \leq T}\left\|u-u_{h}\right\|_{1} \leq C h^{2} \\
& \max _{0 \leq t \leq T}\left\|u-u_{h}\right\| \leq C h^{2.5} .
\end{aligned}
$$

\section{NuMERICAL EXPERIMENTS}

In this section we present the results of some numerical experiments that we performed to approximate solutions of the Camassa-Holm equation, in its reduced form RCH (i.e. with $k=0$ in (1.1)). using the Galerkin finite element methods analyzed in the previous sections to discretize the equation in the spatial variable. In various places in the sequel use will be made of formulas of exact smooth travelling-wave solutions of RCH in the notation of [39] (see specifically formulas (2.29)-(2.30) in that reference): Let $\kappa>0, p>0$, be two parameters such that $0<\kappa p<1$, and $\tilde{c}=2 \kappa^{2} /\left(1-\kappa^{2} p^{2}\right), V=\tilde{c}+\kappa^{2}$. Then for $x_{0} \in \mathbb{R}$ the formulas

$$
u(\xi)=\kappa^{2}+\frac{\tilde{c}^{2} p^{2} \operatorname{sech}^{2} \frac{1}{2} \theta}{2+\tilde{c} p^{2} \operatorname{sech}^{2} \frac{1}{2} \theta},
$$

with

$$
\xi=x-V t+x_{0}=\frac{\theta}{\kappa p}+\ln \left[\frac{(1+\kappa p)+(1-\kappa p) e^{\theta}}{(1-\kappa p)+(1+\kappa p) e^{\theta}}\right]
$$

define parametrically a family of single-pulse travelling-wave solutions of $(\mathrm{RCH})$ that are centered at $x=x_{0}$, travel to the right with speed $V$ and decay exponentially as $|x| \rightarrow \infty$ to $\kappa^{2}$. (As a consequence of the changeof-variable formula that transforms (1.1) into the $\mathrm{RCH}$ (1.4), it is seen that (1.1) with $k=\kappa^{2}$ possesses smooth solitary-wave solutions decaying to zero as $|x| \rightarrow \infty$, and found by replacing $u$ in (6.1) by $u+\kappa^{2}$ and putting $V=\tilde{c}$.) As $\kappa \rightarrow 0$ with $\kappa p \rightarrow 1$, the solution (6.1)-(6.2) of the RCH tends to the one-parameter family of peakon (soliton) solutions of the $\mathrm{RCH}$ given explicitly by

$$
u(x, t)=\tilde{c} \exp \left(-\left|x-\tilde{c} t+x_{0}\right|\right) .
$$

(The corresponding non-smooth travelling wave solutions of $\mathrm{CH}$ with $k \neq 0$ are given by $u=\tilde{u}-k$, where $\tilde{u}=\tilde{c} \exp \left(-\left|x-(\tilde{c}-k) t+x_{0}\right|\right)$. In the numerical experiments we compute numerically the solution (6.1) (6.2) for each $(x, t)$, given the parameters $\kappa, p$, by solving (6.2) for $\theta$ by Newton's method with absolute error tolerance $10^{-10}$ and substituting in (6.1).

6.1. Fully discrete schemes and stability conditions. We used the classical, explicit, four-stage, fourthorder accurate Runge-Kutta scheme with a uniform time step (RK4) for the temporal discretization of the o.d.e. systems representing the various space-discrete finite element schemes of the previous sections. (Unless otherwise mentioned, we solved numerically the periodic initial-value problem for RCH.) The integrals in the finite element equations were approximated by Gauss-Legendre quadrature with five nodes in each mesh interval for cubic and quadratic splines and with three nodes for piecewise linear discretizations. The initial 
conditions of the fully discrete scheme were normally taken as the 'elliptic' $\left(H^{1}\right)$ projections of the initial data; taking the $L^{2}$ projections gave practically the same results.

Due to the presence of the $-u_{x x t}$ term in the left-hand side of RCH the o.d.e. semidiscrete systems are only mildly stiff and the fully discrete schemes were stable under a Courant number restriction. To get some indication of the maximum allowed Courant number we approximated two smooth travelling wave solutions given by (6.1) - (6.2) corresponding to $\kappa=1$ and $V=4$, and $V=6$, on $[-100,100]$ with $h=0.1$, and tested the stability of the code integrating up to $T=100$. We also studied the stability of the code for peakons given by (6.3) for $V=\tilde{c}=1,2,3$ on $[-100,100]$ with $h=0.05$. The maximum allowed values of the Courant number $V \Delta t / h$ for stability are recorded in Table 1. They depend on $V$ in the case of smooth travelling waves but are independent of $V$ for peakons.

\begin{tabular}{c||cc|ccc}
\hline \multicolumn{2}{c}{ Standard Galerkin } & \multicolumn{3}{c}{ Modified Galerkin } \\
\hline Travelling wave $V=4$ & 2.92 & 3.91 & 2.62 & 2.93 & 3.93 \\
Travelling wave $V=6$ & 2.18 & 2.68 & 1.98 & 2.18 & 1.79 \\
peakons & 1.54 & 1.83 & 1.41 & 1.54 & 1.83 \\
\hline
\end{tabular}

TABLE 1. Maximum Courant number $V \Delta t / h$ for stability for various spatial discretizations

6.2. Spatial convergence rates. We performed a series of numerical experiments with our fully discrete schemes in order to compute the numerical rates of convergence of the spatial discretizations in various norms. (In all cases we checked that the errors practically did not change when we computed with smaller values of $\Delta t$. Usually we took $\Delta t / h=1 / 10$.)

In the case of smooth solutions of the periodic ivp the numerical experiments confirm the theoretical $O\left(h^{r}\right) L^{2}$-error convergence rates. (We experimented, on uniform meshes, with cubic and quadratic splines for the standard Galerkin method, and with cubic, quadratic, and linear splines for the modified scheme). The numerical values of the convergence rates in other norms were practically equal those expected from the approximation properties of the finite element spaces, namely $r-1$ for the $H^{1}$ norm in all cases, $r$ for the $L^{\infty}$ norm in all cases, and $r-2$ in the case of the $H^{2}$ norm for cubic and quadratic splines. It is noteworthy that for the same test problem the values of the two spatial discretizations (standard and modified) were very close in all norms.

In order to find the numerical convergence rates in the case of nonsmooth solutions we computed the evolution of the peakon of unit speed $u(x, t)=\exp (-|x-t|)$ on the interval $[-40,40]$ with periodic boundary conditions, up to $T=1$, using uniform meshes in space with $h=80 / N$ and $\Delta t / h=1 / 10, \Delta t=1 / M$. We used cubic, quadratic, and linear splines. Table 2 shows the observed errors (normalized by the corresponding norm of the exact solution at $T=1$ ) and convergence rates in the $L^{2}$ and $H^{1}$ norms in the case of cubic splines at $T=1$.

\begin{tabular}{cc||cccc|cccc}
\hline \multicolumn{9}{c}{ Standard Galerkin } & \multicolumn{4}{c}{ Modified Galerkin } \\
\hline$N$ & $M$ & $L^{2}$ error & rate & $H^{1}$ error & rate & $L^{2}$ error & rate & $H^{1}$ error & rate \\
\hline 160 & 20 & $1.1109 \times 10^{-1}$ & - & $4.1633 \times 10^{-1}$ & - & $1.0346 \times 10^{-1}$ & - & $4.0152 \times 10^{-1}$ & - \\
320 & 40 & $5.1323 \times 10^{-2}$ & 1.114 & $3.1138 \times 10^{-1}$ & 0.419 & $4.6734 \times 10^{-2}$ & 1.147 & $2.9610 \times 10^{-1}$ & 0.439 \\
640 & 80 & $2.3124 \times 10^{-2}$ & 1.150 & $2.3106 \times 10^{-1}$ & 0.430 & $2.0617 \times 10^{-2}$ & 1.181 & $2.1716 \times 10^{-1}$ & 0.447 \\
1280 & 160 & $1.0417 \times 10^{-2}$ & 1.150 & $1.7091 \times 10^{-1}$ & 0.435 & $9.1382 \times 10^{-3}$ & 1.174 & $1.5881 \times 10^{-1}$ & 0.451 \\
2560 & 320 & $4.7544 \times 10^{-3}$ & 1.132 & $1.2626 \times 10^{-1}$ & 0.437 & $4.1283 \times 10^{-3}$ & 1.146 & $1.1600 \times 10^{-1}$ & 0.453 \\
5120 & 640 & $2.2090 \times 10^{-3}$ & 1.106 & $9.3242 \times 10^{-2}$ & 0.437 & $1.9097 \times 10^{-3}$ & 1.112 & $8.4706 \times 10^{-2}$ & 0.454 \\
\hline
\end{tabular}

TABLE 2. $L^{2}$ and $H^{1}$ normalized errors and convergence rates at $T=1$, peakon solution, cubic splines, $h=80 / N, \Delta t=1 / M$

The experimental rates of the $L^{2}$ errors are equal to about 1.1 for both Galerkin methods and those of the $H^{1}$ errors between 0.4 and 0.5 . The errors of the modified method are slightly smaller. In Table 3 we compare the normalized $L^{2}, L^{\infty}$, and $H^{1}$ errors and convergence rates of the cubic $(r=4)$, quadratics 
$(r=3)$, and linear $(r=2)$ spline discretizations for the same test problem. We show only the data for the line corresponding to $N=5120, M=640$ ). As expected from the low regularity of the solution, the rates of convergence for all spline discretizations were roughly the same for a given norm. It appears that the modified scheme with cubic splines yields the smallest errors.

\begin{tabular}{l||ll|lll}
\hline \multicolumn{3}{c}{ Standard Galerkin } & \multicolumn{3}{c}{ Modified Galerkin } \\
\hline Norm & $r=4$ & $r=3$ & $r=4$ & $r=3$ & $r=2$ \\
\hline$L^{2}$ error & $2.2090 \times 10^{-3}$ & $3.3557 \times 10^{-3}$ & $1.9097 \times 10^{-3}$ & $2.6936 \times 10^{-3}$ & $3.3828 \times 10^{-3}$ \\
rate & 1.106 & 1.064 & 1.112 & 1.060 & 1.125 \\
$L^{\infty}$ error & $7.2834 \times 10^{-3}$ & $1.1634 \times 10^{-2}$ & $6.5729 \times 10^{-3}$ & $7.9459 \times 10^{-3}$ & $1.3519 \times 10^{-2}$ \\
rate & 0.902 & 0.798 & 0.941 & 0.848 & 0.814 \\
$H^{1}$ error & $9.3241 \times 10^{-2}$ & $1.0899 \times 10^{-1}$ & $8.4706 \times 10^{-2}$ & $9.0104 \times 10^{-2}$ & $1.1564 \times 10^{-1}$ \\
rate & 0.437 & 0.403 & 0.454 & 0.443 & 0.407 \\
\hline
\end{tabular}

TABLE 3. $L^{2}, L^{\infty}$, and $H^{1}$ normalized errors and convergence rates at $T=1$, peakon solutions, splines of order $r, N=80 / h=5120, M=1 / \Delta t=640$

We also discretized the ibvp (C-H-s-b) for the $\mathrm{RCH}$ using the spatial discretization (5.8)-(5.10) coupled with RK4 in order to check the theoretically predicted convergence rates of the error bounds in (5.11) and (5.25). The spatial discretization was effected using piecewise linear continuous functions $(r=2)$ and cubic splines $(r=4)$ on uniform and nonuniform meshes in several test problems. In the case of uniform mesh the $L^{2}, L^{\infty}$, and $H^{1}$ numerical convergence rates were found to be approximately equal to $r, r$, and $r-1$, respectively, for the approximations of both $m$ and $u$ in (5.11) and (5.25). Thus, the predicted rates of the $L^{2}$ errors for $\mathrm{m}$ and $\mathrm{u}$ in (5.11) and (5.25) appear to be pessimistic for uniform meshes. In the case of a quasiuniform mesh (we experimented with the mesh $h / 2,3 h / 2, h / 2,3 h / 2, \cdots, h=1 / N$ ), some results are shown in Table 4 for a test example with appropriate right-hand side and exact solution $u(x, t)=e^{t}\left[x \sin \pi x-\frac{\pi}{6}(x-1 / 2)+\frac{2 \pi}{3}(x-1 / 3)^{3}\right], m=u-u_{x x}$. In the case of piecewise linear functions the table shows that the apparent $L^{2}$-convergence rates are equal to 1 for the approximation of $m$ and 2 for that of $u$. (The $L^{\infty}$ rates, not shown in the table, were approximately equal to 1 for $m$ and 2 for $u$, while the $H^{1}$ rate for $u$ was equal to 1.) For cubic splines the observed $L^{2}$ rates were approximately equal to 3 and 4 for $m$ and $u$, respectively. (The $L^{\infty}$ and $H^{1}$ rates for $m$ were equal to approximately 2.8 and 2, respectively, whereas the corresponding rates for $u$ came out to be 4 and 3, respectively.) Thus, it appears that for our

(a) $r=2$

\begin{tabular}{c||cc|cc}
\hline \multicolumn{5}{c}{$m$} \\
\hline$N$ & $L^{2}$ error & rate & $L^{2}$ error & rate \\
\hline 32 & $7.2147 \times 10^{-1}$ & - & $1.2979 \times 10^{-3}$ & - \\
64 & $3.9516 \times 10^{-1}$ & 0.866 & $3.2705 \times 10^{-4}$ & 1.989 \\
128 & $2.1177 \times 10^{-1}$ & 0.900 & $8.1585 \times 10^{-5}$ & 2.003 \\
256 & $1.0508 \times 10^{-1}$ & 1.011 & $2.0389 \times 10^{-5}$ & 2.000 \\
512 & $5.2300 \times 10^{-2}$ & 1.007 & $5.0977 \times 10^{-6}$ & 2.000 \\
1024 & $2.6110 \times 10^{-2}$ & 1.002 & $1.2745 \times 10^{-6}$ & 2.000 \\
2048 & $1.3048 \times 10^{-2}$ & 1.001 & $3.1862 \times 10^{-7}$ & 2.000 \\
\hline
\end{tabular}

(b) $r=4$

\begin{tabular}{c||cc|cc}
\hline \multicolumn{5}{c}{$m$} \\
\hline$N$ & $L^{2}$ error & rate & $L^{2}$ error & rate \\
\hline 8 & $3.9055 \times 10^{-2}$ & - & $3.8715 \times 10^{-4}$ & - \\
16 & $6.0719 \times 10^{-3}$ & 2.685 & $3.4546 \times 10^{-5}$ & 3,486 \\
32 & $8.2945 \times 10^{-4}$ & 2.872 & $2.5099 \times 10^{-6}$ & 3.783 \\
64 & $1.0748 \times 10^{-4}$ & 2.948 & $1.6634 \times 10^{-7}$ & 3.915 \\
128 & $1.3568 \times 10^{-5}$ & 2.986 & $1.0654 \times 10^{-8}$ & 3.965 \\
256 & $1.7037 \times 10^{-6}$ & 2.993 & $6.7326 \times 10^{-10}$ & 3.984 \\
512 & $2.1325 \times 10^{-7}$ & 2.998 & $4.2300 \times 10^{-11}$ & 3.992 \\
1024 & $2.6665 \times 10^{-8}$ & 3.000 & $2.7035 \times 10^{-12}$ & 3.968 \\
\hline
\end{tabular}

TABLE 4. $L^{2}$ errors and rates of convergence for $m$ and $u$ for the ibvp (CH-s-b), quasiuniform mesh, (a) linear splines, (b) cubic splines

quasiuniform mesh examples the rates in (5.11) are sharp, while the rate $r-1 / 2$ in (5.25) is pessimistic by $1 / 2$. (The reduction of order for the Galerkin approximation of $m$ is not surprising since $m$ is the solution of a first-order hyperbolic p.d.e.; the $L^{2}$ rate of the approximation of $u$ is expected to be optimal as $u_{h}$ is the Galerkin approximation of a two-point b.v.p. whose right-hand side $m_{h}$ is $C^{0}$ if $r=2$ and $C^{2}$ if $r=4$.) 
6.3. Conservation of invariants. It is well known, cf. [7, and easy to check, that solutions of the Cauchy problem of the $\mathrm{CH}$ equation (1.1) and also of the periodic ivp for (1.1) on an interval $(a, b)$ satisfy the conservation laws $\frac{d}{d t} H_{i}[u]=0, i=0,1,2$, where

$$
H_{0}[u]=\int_{a}^{b} u d x, \quad H_{1}[u]=\int_{a}^{b}\left(u^{2}+u_{x}^{2}\right) d x, \quad H_{2}[u]=\int_{a}^{b} u\left(u^{2}+u_{x}^{2}+2 k u\right) d x .
$$

In the case of the periodic ivp for the $\mathrm{CH}$ in the system form (1.3) it holds that $\frac{d}{d t} \tilde{H}_{i}[m, u]=0, i=0,1,2$, where

$$
\tilde{H}_{0}[m, u]=\int_{a}^{b} m d x, \quad \tilde{H}_{1}[m, u]=\int_{a}^{b} m u d x, \quad \tilde{H}_{2}[m, u]=\int_{a}^{b}\left(u^{2} m-u u_{x}^{2}+2 k u^{2}\right) d x .
$$

In order to assess the quality of the numerical approximations, it is important to check the extent to which the fully discrete schemes under study preserve these invariants. (As usual, we consider the RCH equation). The standard Galerkin semidiscretization (3.1) clearly preserves $H_{0}$ and $H_{1}$ in the sense that $H_{i}\left[u_{h}(t)\right]=H_{i}\left[u_{h}(0)\right], i=0,1$. The invariant $H_{0}$ is also trivially preserved by the full discretization of (3.1) by the RK4 scheme, in contrast to $H_{1}$ which is not preserved. The modified Galerkin method (4.1)-(4.2) for the periodic ivp preserves $\tilde{H}_{1}$ : Let $m_{h}, u_{h}$ be the solution of (4.1)-(4.4). Then, using (4.2) with $\phi=u_{h}$, and (4.1) with $\phi=u_{h t}$ we have

$$
\begin{aligned}
\frac{d}{d t} \tilde{H}_{1}\left[m_{h}, u_{h}\right] & =\left(m_{h t}, u_{h}\right)+\left(m_{h}, u_{h t}\right) \\
& =-\left(\left(m_{h} u_{h}\right)_{x}, u_{h}\right)-\left(m_{h} u_{h x}, u_{h}\right)+\left(u_{h}, u_{h t}\right)+\left(u_{h x}, u_{h x t}\right) \\
& =\frac{1}{2} \frac{d}{d t}\left\|u_{h}\right\|_{1}^{2} .
\end{aligned}
$$

On the other hand (4.1) with $\phi=u_{h}$ gives $\left(m_{h}, u_{h}\right)=\left\|u_{h}\right\|_{1}^{2}$, i.e. $\frac{d}{d t} \tilde{H}_{1}\left[m_{h}, u_{h}\right]=\frac{d}{d t}\left\|u_{h}\right\|_{1}^{2}$. From these identities we conclude that $\frac{d}{d t} \tilde{H}_{1}\left[m_{h}, u_{h}\right]=\frac{d}{d t}\left\|u_{h}\right\|_{1}^{2}=0$, as claimed. Note that the temporal discretization of (4.1)-(4.4) with RK4 does not conserve $\tilde{H}_{1}$.

In what follows we present some graphs of the temporal evolution of the invariants using the fully discrete schemes. We computed the quantities

$$
E_{i}(t)=\log _{10}\left|\frac{H_{i}(t)-H_{i}(0)}{H_{i}(t)}\right|
$$

(and the analogous $\tilde{H}_{i}$ in the case of the modified method) for $t \geq \Delta t$. Here, $H_{i}(t)=H_{i}\left(u_{h}(t)\right.$ ) and $\tilde{H}_{i}(t)=\tilde{H}_{i}\left[m_{h}(t), u_{h}(t)\right]$. In the case of the smooth solutions we solved numerically the periodic ivp for the $\mathrm{RCH}$ on the spatial interval $[-50,50]$ up to $t=100$ with the standard and modified method using as initial condition $u_{0}(x)=1+e^{-x^{2}}$.

Figure 1 shows the graphs of the logarithmic relative errors $E_{i}$ of $H_{i}$ as functions of $t$ for this example, produced for $h=0.1$ and diminishing $\Delta t / h$ by the standard Galerkin method with cubic splines coupled with RK4 time stepping. $H_{0}$ is preserved to roundoff as expected, while $H_{2}$ is preserved to about 8 decimal digits for this value of $h$. (The error in $H_{2}$ is practically due to the spatial approximation since it remains almost constant as $\Delta t$ is decreased.) The error in $H_{1}$ also diminishes with $\Delta t$; for $\frac{\Delta t}{h}=\frac{1}{200}$ its temporal discretization part is practically negligible and $H_{1}$ is preserved to roundoff as expected.

In the case of the modified Galerkin fully discrete scheme the cubic spline results for the preservation of the $H_{i}=H_{i}\left[u_{h}\right]$ are practically the same with those shown in Figure 1. In Figure 2 we show the evolution of the logarithmic errors $\tilde{E}_{i}$ of the invariants $\tilde{H}_{i}\left[m_{h}, u_{h}\right]$ pertinent to the system form of RCH for the fully discrete schemes with cubic splines and piecewise linear functions (the latter are admitted by the modified scheme), for the same example, with $h=0.1$ and $\Delta t=10^{-3}$. For cubic splines $\tilde{H}_{2}$ is preserved to at least 7 decimal digits and to about 5 digits for the less accurate discretization with piecewise linear functions. For both discretizations the relative error of $\tilde{H}_{1}$ is of $O\left(10^{-13}\right)$ and is due to the temporal discretization as $\tilde{H}_{1}$ is preserved for both semidiscrete schemes. Apparently $\tilde{H}_{0}$ seems to be preserved to almost roundoff in both cases. In the case of peakons we computed the logarithmic errors of the invariants as functions of $t$ in several test runs. In Figure 3, as an example, we show the results in the case of a peakon of unit amplitude and speed approximated by the standard Galerkin method with cubic splines for our usual (a), and a finer 

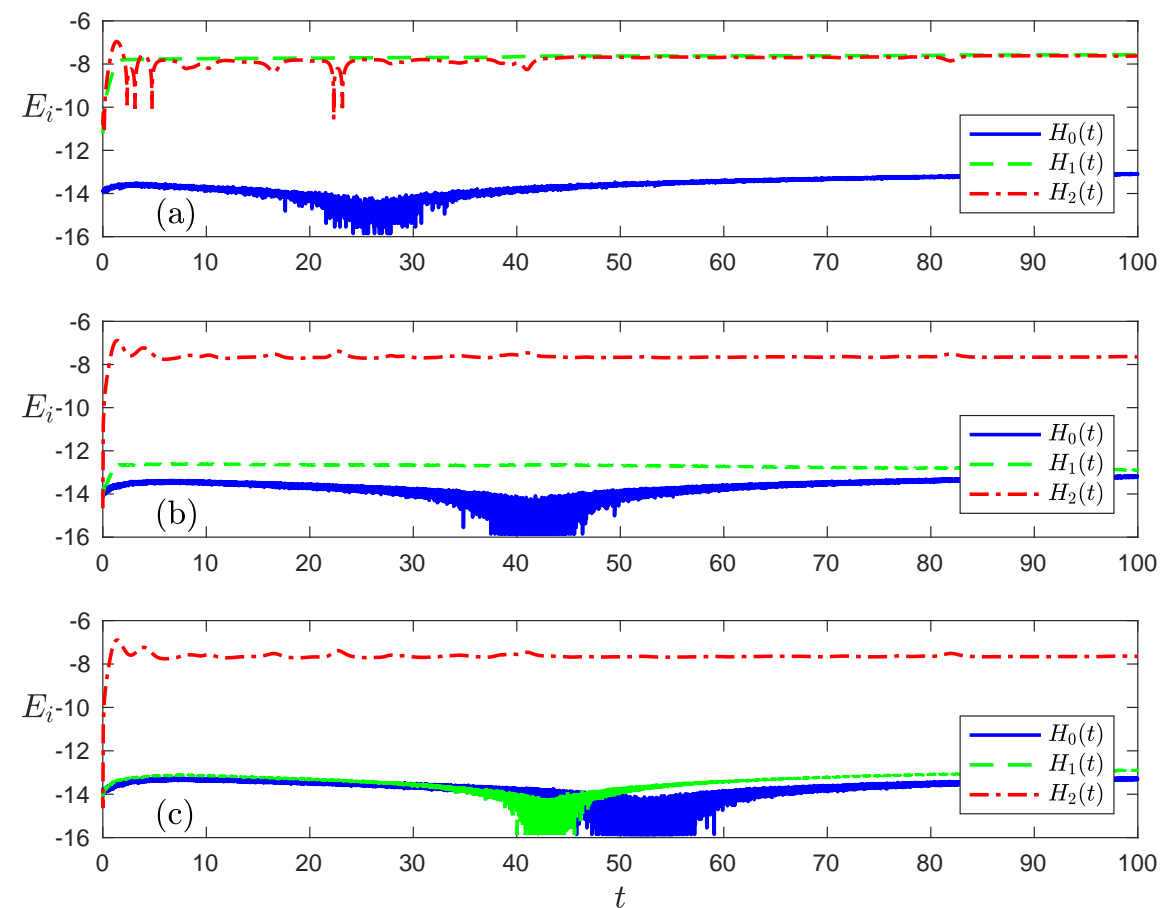

Figure 1. Preservation of invariants $H_{0}, H_{1}, H_{2}$, smooth solutions. Standard Galerkin method with cubic splines, $h=0.1$, (a) $\frac{\Delta t}{h}=\frac{1}{10}$, (b) $\frac{\Delta t}{h}=\frac{1}{100}$, (c) $\frac{\Delta t}{h}=\frac{1}{200}$
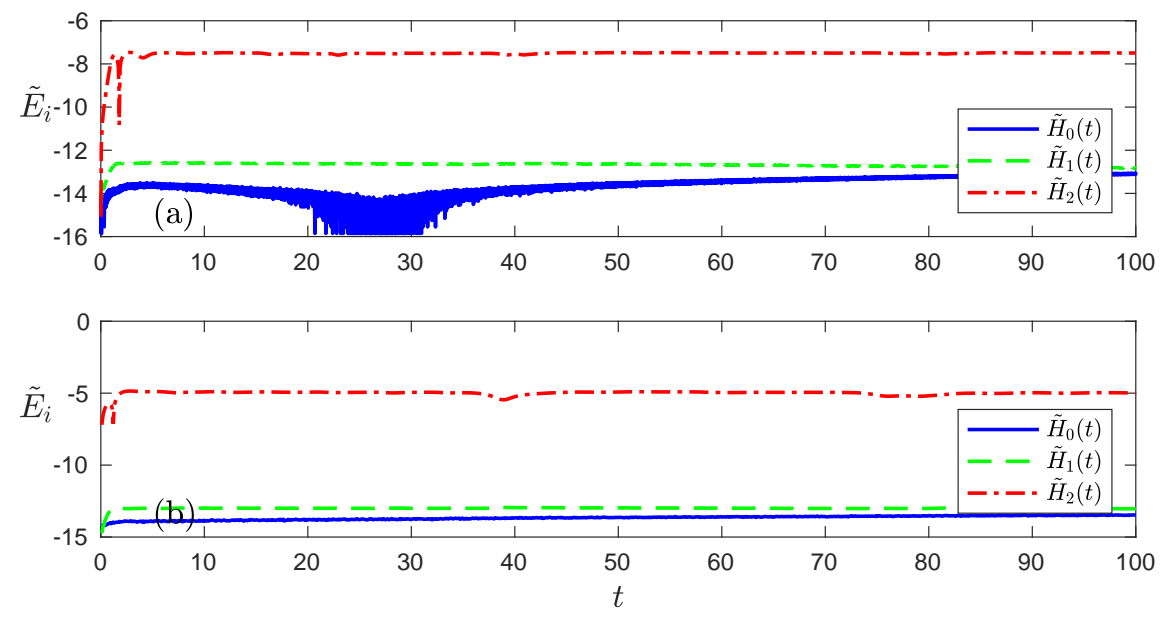

FiguRE 2. Preservation of invariants $\tilde{H}_{0}, \tilde{H}_{1}, \tilde{H}_{2}$, smooth solutions. modified Galerkin method, $h=0.1, \Delta t=10^{-3}$ (a) cubic splines, (b) p.w. linear continuous functions

mesh (b). We observe that $H_{0}$ is preserved to roundoff, that the error of $H_{1}$ is small and is reduced by at most two digits in (b), while that of $H_{2}$ practically remains the same. This is due of course to the lack of smoothness of the solution that reduces the rate of convergence of the schemes, cf. Section 6.2 .

6.4. Error indicators for travelling waves. In order to further assess the accuracy of our numerical methods we studied several error indicators pertinent to travelling-wave solutions, namely the amplitude, phase, speed, and shape errors, [5], 4, for smooth travelling waves and peakons. Since the amplitude of the 

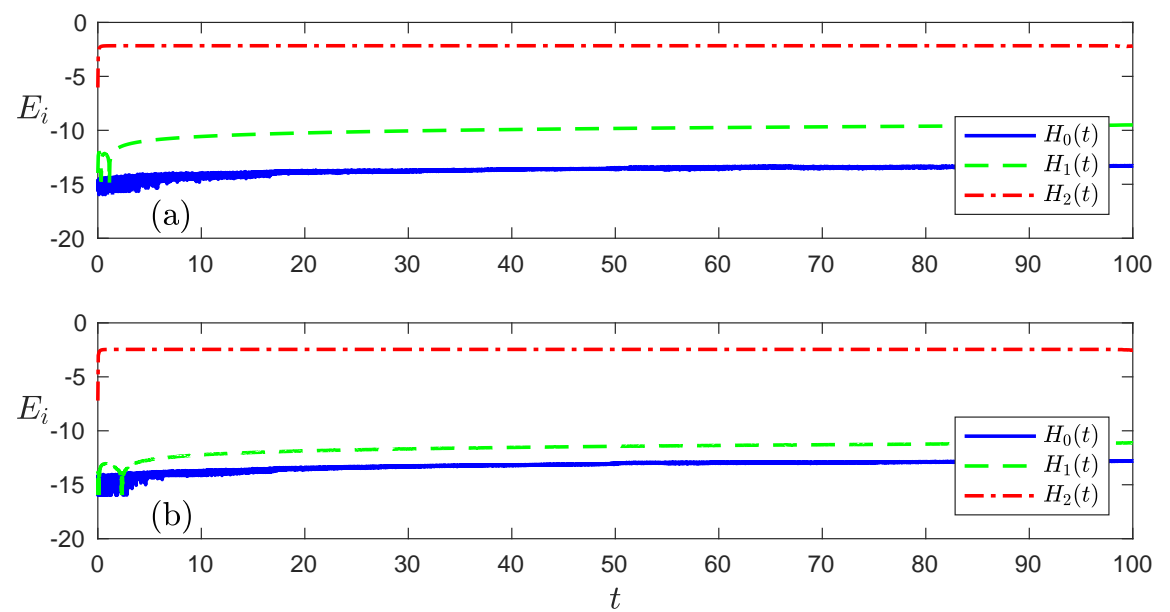

Figure 3. Preservation of invariants $H_{0}, H_{1}, H_{2}$, peakon, $A=1$. Standard Galerkin method with cubic splines, (a) $h=0.1, \Delta t=10^{-3}$, (b) $h=0.05, \Delta t=2.5 \times 10^{-4}$

travelling wave remains constant, we define the (normalized) amplitude error at $t=t^{n}=n \Delta t$ as

$$
E_{a m p}\left(t^{n}\right)=\frac{\left|U\left(x^{*}\left(t^{n}\right), t^{n}\right)-U_{0}\right|}{\left|U_{0}\right|},
$$

where $U\left(x, t^{n}\right)$ is the fully discrete numerical approximation at $t^{n}, U_{0}$ is the initial amplitude (peak value) of the exact travelling wave and $x^{*}\left(t^{n}\right)$ is the point where $U\left(\cdot, t^{n}\right)$ achieves its maximum. The value of $x^{*}\left(t^{n}\right)$ is found by solving the equation $\frac{d}{d x} U\left(x, t^{n}\right)=0$ by Newton's method (with tolerance $10^{-10}$ ) for smooth solutions and by the bisection method for peakons. (For piecewise linear functions we took as $x^{*}$ the mesh point where $\max _{i} U\left(x_{i}, t^{n}\right)$ occurs.) As initial value for the Newton iteration we took the quadrature node at which $U$ has a discrete maximum. We started the bisection method by taking an interval of length $4 h$ around that quadrature node.

Finding $x^{*}\left(t^{n}\right)$ enables us to compute a phase error of the numerical solution $U$ at $t^{n}$ as

$$
E_{\text {phase }}\left(t^{n}\right)=\left|x^{*}\left(t^{n}\right)-V t^{n}\right|,
$$

for a travelling wave of speed $V$, initially centered at $x=0$, and a speed error defined as

$$
E_{\text {speed }}\left(t^{n}\right)=\left|V-V_{h}\left(t^{n}, \tau\right)\right|,
$$

where $V_{h}\left(t^{n}, \tau\right)=\left(x^{*}\left(t^{n}\right)-x^{*}\left(t^{n}-\tau\right)\right) / \tau$. We usually take $\tau$ much larger than $\Delta t$ to smooth out oscillations in the discrete speed $V_{h}\left(t^{n}, \tau\right)$. (In the computations to be reported in the sequel on the temporal interval $[0,100]$, we normally take $\tau=1$.)

Finally, the (normalized) shape error measures the amount by which the numerical solution at $t^{n}$ differs from the exact travelling wave profile $u\left(\cdot, t^{n}\right)$ traslated so as to give the best fit. It is defined with respect to the $L^{2}$ norm as

$$
E_{\text {shape }}\left(t^{n}\right)=\min _{s} \zeta(s), \quad \zeta(s):=\frac{\left\|U\left(\cdot, t^{n}\right)-u(\cdot, s)\right\|}{\|u(\cdot, 0)\|},
$$

where the minimum of $\zeta(s)$ is found again using the Newton or the bisection method to solve $\frac{d}{d s} \zeta^{2}(s)=0$ in the vicinity of $t^{n}$.

In Table 5 we show the values of the amplitude, phase and shape error at $t^{n}=100$ of the fully discrete approximations produced by the standard and the modified Galerkin method in the case of a smooth travelling wave of the form (6.1) - (6.2) with $\kappa=1$ and $V=4.333$. We took the spatial interval $[-100,100]$ and computed with $h=0.1,0.05,0.025$ and $\Delta t=h / 10$.

As expected, cubic splines $(r=4)$ give the best results, while there is not much difference between the standard and the modified methods for $r=4$ and $r=3$. Over time, all these errors oscillate somewhat about mean values that remain practically constant in $t$ for $h=0.05$ and $h=0.025$. 
(a) Standard Galerkin

\begin{tabular}{|c|c|c|c|c|c|c|}
\hline \multicolumn{4}{|c|}{$r=4$, cubic splines } & \multicolumn{3}{|c|}{$r=3$, quadratic splines } \\
\hline$h$ & $E_{a m p}$ & $E_{\text {phase }}$ & $E_{\text {shape }}$ & $E_{a m p}$ & $E_{\text {phase }}$ & $E_{\text {shape }}$ \\
\hline 0.1 & $9.1617 \times 10^{-9}$ & $7.0771 \times 10^{-6}$ & $1.2058 \times 10^{-8}$ & $5.4368 \times 10^{-7}$ & $2.6859 \times 10^{-5}$ & $1.0699 \times 10^{-7}$ \\
\hline 0.05 & $5.5416 \times 10^{-10}$ & $3.1641 \times 10^{-7}$ & $5.6834 \times 10^{-10}$ & $3.2712 \times 10^{-8}$ & $1.5455 \times 10^{-6}$ & $6.6264 \times 10^{-9}$ \\
\hline 0.025 & $6.7388 \times 10^{-11}$ & $1.6421 \times 10^{-8}$ & $3.5359 \times 10^{-11}$ & $2.0090 \times 10^{-9}$ & $9.0883 \times 10^{-8}$ & $4.1343 \times 10^{-10}$ \\
\hline \multicolumn{7}{|c|}{ (b) Modified Galerkin } \\
\hline \multicolumn{4}{|c|}{$r=4$, cubic splines } & \multicolumn{3}{|c|}{$r=3$, quadratic splines } \\
\hline$h$ & $E_{a m p}$ & $E_{\text {phase }}$ & $E_{\text {shape }}$ & $E_{a m p}$ & $E_{\text {phase }}$ & $E_{\text {shape }}$ \\
\hline 0.1 & $8.6377 \times 10^{-9}$ & $7.0627 \times 10^{-6}$ & $1.2004 \times 10^{-8}$ & $2.6626 \times 10^{-7}$ & $1.2173 \times 10^{-5}$ & $2.9430 \times 10^{-7}$ \\
\hline 0.05 & $5.5280 \times 10^{-10}$ & $3.1597 \times 10^{-7}$ & $5.6723 \times 10^{-10}$ & $1.5428 \times 10^{-8}$ & $6.3028 \times 10^{-7}$ & $1.7453 \times 10^{-9}$ \\
\hline 0.025 & $7.1619 \times 10^{-11}$ & $1.6134 \times 10^{-8}$ & $3.5361 \times 10^{-11}$ & $9.2885 \times 10^{-10}$ & $3.5562 \times 10^{-8}$ & $1.0862 \times 10^{-10}$ \\
\hline
\end{tabular}

\begin{tabular}{l||lll}
\multicolumn{4}{c}{$r=2$, linear splines } \\
\hline$h$ & $E_{a m p}$ & $E_{\text {phase }}$ & $E_{\text {shape }}$ \\
\hline 0.1 & $4.0487 \times 10^{-5}$ & $1.7600 \times 10^{-3}$ & $6.7965 \times 10^{-5}$ \\
0.05 & $2.7453 \times 10^{-6}$ & $3.3000 \times 10^{-3}$ & $1.6249 \times 10^{-5}$ \\
0.025 & $2.2631 \times 10^{-7}$ & $9.5238 \times 10^{-4}$ & $4.0635 \times 10^{-6}$ \\
\hline
\end{tabular}

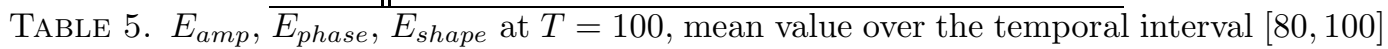
for linear splines $E_{\text {phase }}$ (a) standard Galerkin, (b) modified Galerkin, smooth travelling wave, $\kappa=1, V=4.333$

The speed errors were practically the same for both Galerkin methods with cubic and quadratic splines for the same value of $h$ and diminished of course with $h$. The number of conserved digits of the speed was equal to five for $h=0.1$, six for $h=0.05$ and seven for $h=0.025$. In the case of piecewise linear functions with the modified method the numerical speed preserved one correct digit for $h=0.1$ and two for $h=0.05$ and $h=0.025$.

In Table 6] we show the analogous errors produced by the numerical approximations with the modified method of a peakon of speed $V=1.333$. We took $h=0.05,0.025$ and 0.01 on the interval $[-100,100]$ with $\Delta t=h / 10$ and show the errors at $t^{n}=100$.

\begin{tabular}{l||lll|lll}
\hline \multicolumn{4}{c}{$r=4$, cubic splines } & \multicolumn{3}{c}{$r=3$, quadratic splines } \\
\hline$h$ & $E_{\text {amp }}$ & $E_{\text {phase }}$ & $E_{\text {shape }}$ & $E_{\text {amp }}$ & $E_{\text {phase }}$ & $E_{\text {shape }}$ \\
\hline 0.05 & $1.1717 \times 10^{-2}$ & $6.4696 \times 10^{-1}$ & $2.5744 \times 10^{-2}$ & $1.6177 \times 10^{-2}$ & $1.0482 \times 10^{0}$ & $1.1215 \times 10^{-2}$ \\
0.025 & $6.1999 \times 10^{-3}$ & $3.2055 \times 10^{-1}$ & $1.3246 \times 10^{-2}$ & $8.1867 \times 10^{-3}$ & $5.1723 \times 10^{-1}$ & $6.6000 \times 10^{-3}$ \\
0.01 & $2.6237 \times 10^{-3}$ & $1.2855 \times 10^{-1}$ & $5.9284 \times 10^{-3}$ & $3.4618 \times 10^{-3}$ & $2.0548 \times 10^{-1}$ & $3.2406 \times 10^{-3}$ \\
\hline
\end{tabular}

\begin{tabular}{l||lll}
\multicolumn{4}{c}{$r=2$, linear splines } \\
\hline$h$ & $E_{a m p}$ & $E_{\text {phase }}$ & $E_{\text {shape }}$ \\
\hline 0.05 & $1.1487 \times 10^{-2}$ & $6.5000 \times 10^{-1}$ & $5.8839 \times 10^{-2}$ \\
0.025 & $7.1264 \times 10^{-3}$ & $3.0000 \times 10^{-1}$ & $3.2941 \times 10^{-2}$ \\
0.01 & $3.5565 \times 10^{-3}$ & $1.1000 \times 10^{-1}$ & $1.3585 \times 10^{-2}$ \\
\hline
\end{tabular}

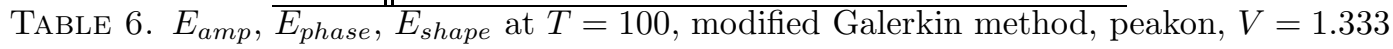

The errors of the standard Galerkin method (for $r=4$ and $r=3$ ) were roughly the same. The errors did not oscillate in time and practically remained constant for the smaller values of $h$. Because the maximum of the the maximum of the peakon for linear splines coincides with the mesh node $x=33.3$ at $T=100$ the actual $E_{\text {phase }}$ happened to be 0 . For this reason we present the mean value of the $E_{\text {phase }}$ for all times $t^{n} \in[80,100]$. In all cases the speed was conserved to two digits when $h=0.05$ and $h=0.025$, and to three digits when $h=0.01$.

6.5. Approximation of the generation and interactions of peakons. In closing we record some observations concerning the approximation of peakons by the fully discrete Galerkin methods under consideration: 
(i) When a nonsmooth function such as a peakon is used as initial value in an evolution numerical experiment one expects a practically localized oscillatory error to appear at $t=0$ as a result of projecting the peakon onto the finite element space. As previously mentioned we normally use as initial value of the discrete schemes the $H^{1}$ projection of the initial data; using the $L^{2}$ projection or the interpolant leads to similar errors.

As an example, consider the evolution shown in Figure 4 in which a peakon of unit speed, initially centered at $x=0$, is approximated by the $H^{1}$ projection in the space, of quadratic splines and its evolution is effected by the fully discrete modified Galerkin method up to $T=50$. An oscillatory error, decaying fast in space, of amplitude $7.5 \times 10^{-3}$ for $h=0.05$, is generated near $x=0$ where the initial peakon was located. It diminishes slowly as $h$ is decreased; for example its amplitude is about $1.6 \times 10^{-3}$ when $h=6.25 \times 10^{-3}$. A similar error is observed in the case of the standard Galerkin method for the other spline spaces; the modified method gives somewhat better results. We observed that the same type of error appears in spectral

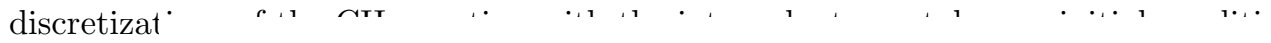
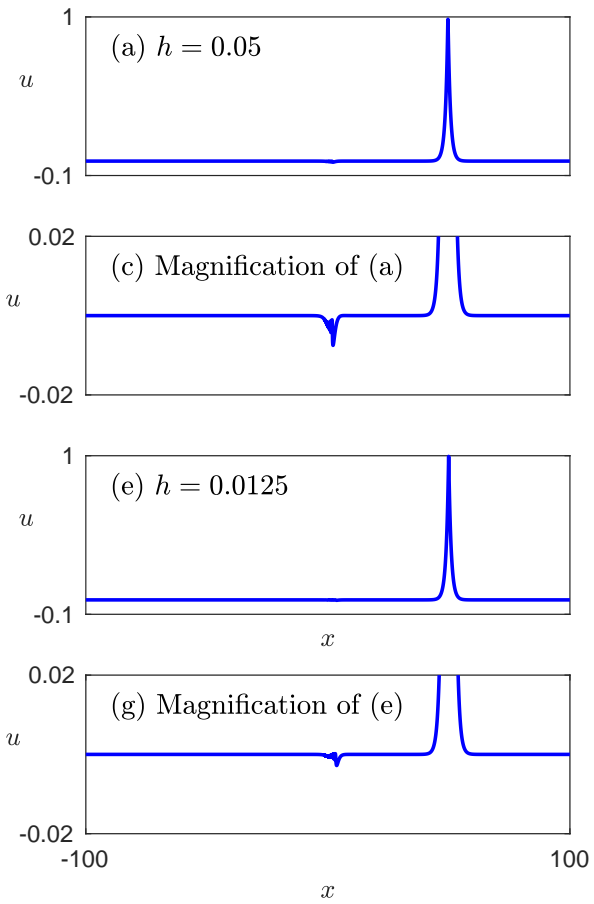
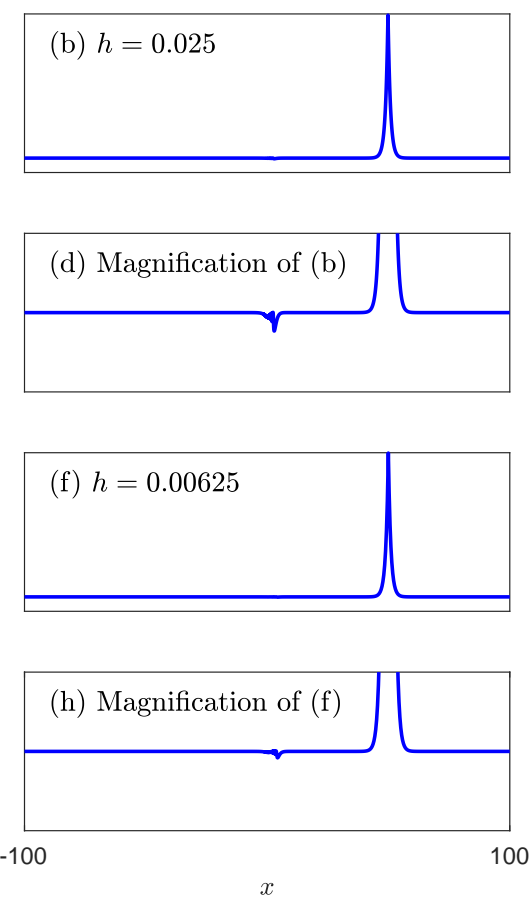

FiguRE 4. Peakon propagation with the modified Galerkin method and quadratic splines for various values of $h, \Delta t=h / 10, T=50$

This small noise remains stationary in time in the vicinity of the spatial point of its generation. Apparently this property is shared by the $\mathrm{CH}$ equation (due to the presence of the $-u_{x x t}$ term) with the BBM equation, for which, it was shown in [3] that initial discontinuities in the data do not propagate. (Similar errors, noticed in numerical solutions of the BBM equation in [5], were caused by the truncation of the decaying ends of an initial solitary-wave profile in that reference.)

(ii) Peakons, being solitons, interact elastically, and their overtaking collisions have been described in detail analytically cf. e.g. [15, 41]. However, when their interactions are simulated by a numerical evolution method one observes that numerical artifacts, in the form of small residual waves, are produced.

As an example, we consider two peakons of amplitudes 1.0 and 0.5 centered initially at $x=-20$ and 20 , respectively. With those initial values we integrate the $\mathrm{RCH}$ with periodic conditions on the interval $[-100,100]$ using the fully discrete modified Galerkin method with cubic splines with $h=5 \times 10^{-3}$ and $\Delta t=h / 10$. The results of the numerical evolution up to $t=200$ are shown in Figure 5 .

In Figure 5(c), at $t=25$, we observe again the small oscillations (stationary in the vicinity of $x=-20$ and $x=20$ ) produced by the $H^{1}$ projection of the initial peakons in the finite element space and previously 

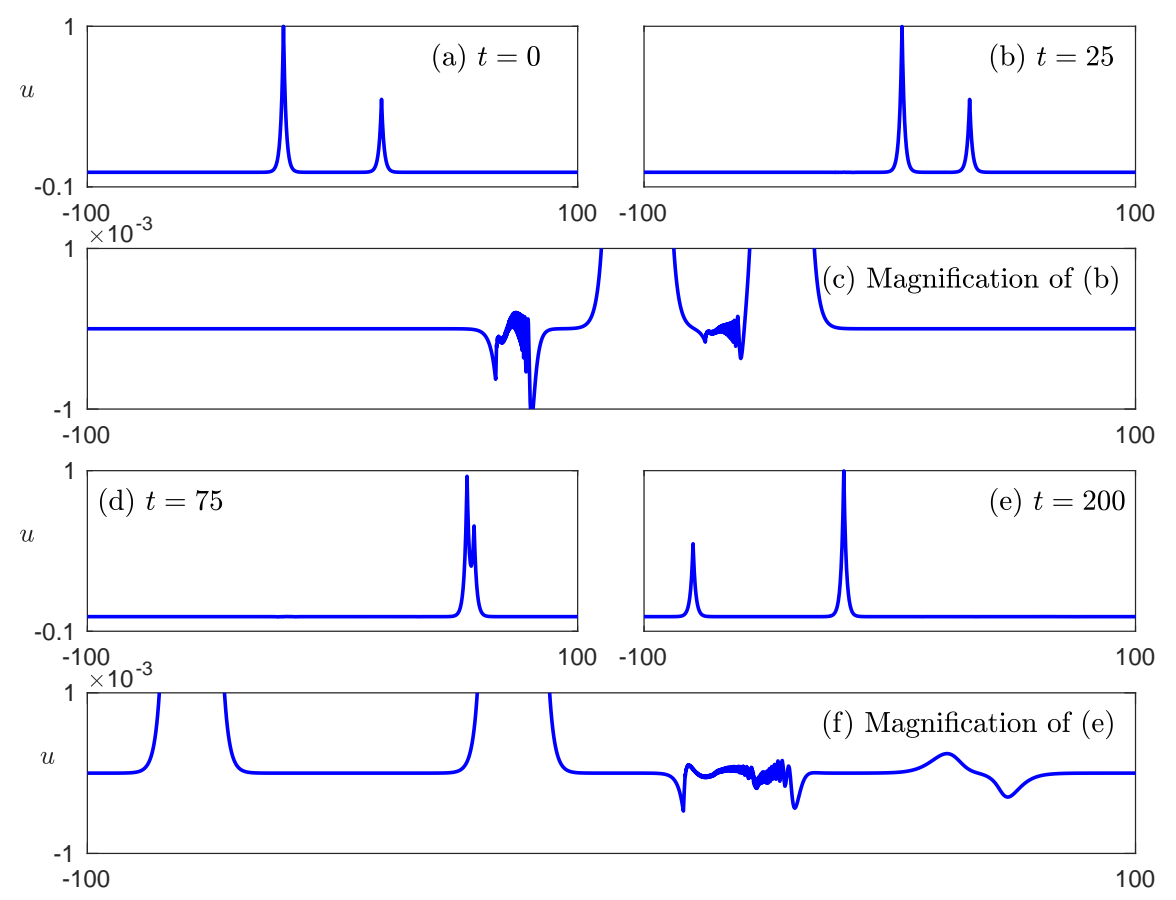

Figure 5. Interaction of two peakons, modified Galerkin, cubic splines, $h=0.005, \Delta t=h / 10$

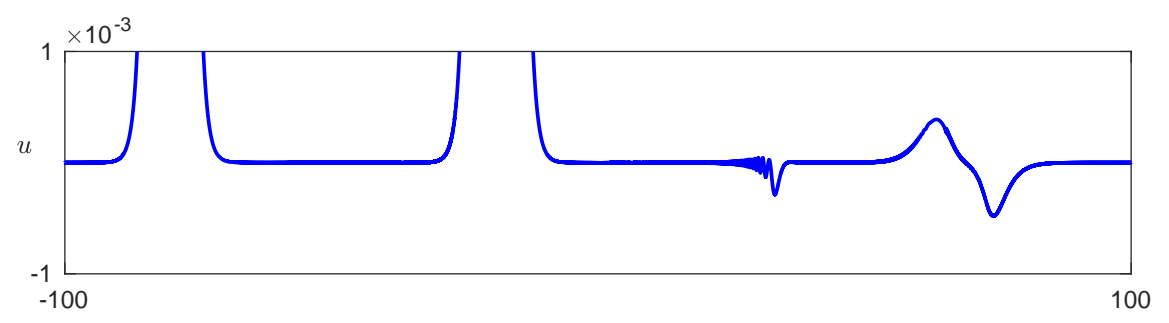

FiguRE 6. Interaction of two peakons (evolution of Figure 5) using the standard pseudospectral-RK4 method, $N=16384, \Delta=5 \times 10^{-3}$

commented on. The peakons first interact in a spatial and temporal window centered at $(x, t)=(60,80)$ and wrap around the boundary due to periodicity. At $t=200$ (Figure 5(e), (f)) we observe the high frequency stationary oscillations produced near $x=20$ due to the initial approximation of the smaller peakon. (The analogous oscillations produced near $x=-20$ by the projection of the larger peakon have 'climbed' on the larger peakon which is centered at $x=-20$ at this time instant.) We also observe near $x=60$ a large-wavelength wavelet of amplitude about $2.8 \times 10^{-4}$. This wavelet is stationary; note that the dispersion relation of the linearized $\mathrm{RCH}$ is $\omega=0$, and therefore small-amplitude disturbances are not expected to propagate. The wavelet is a numerical artifact produced by the numerical approximation of the peakon collision. Its amplitude diminishes slowly as $h$ is decreased; e.g. it was equal to $5 \times 10^{-4}$ when $h=0.01$. The wavelet reappeared near $x=60$ after the next interaction (due to periodicity), of the peakons, that occurred around $t=480$. (In order to observe the wavelet after the subsequent interactions, which all occur near $x=60$, one has to 'clean' smoothly the solution profile well after the first interaction from small-amplitude artifacts and let the main pulses interact again). That the wavelet is specific to the numerical interaction of peakons may also be seen from the fact that it is absent when overtaking collisions of smooth travelling waves of the RCH are simulated numerically. When we did so (with the same numerical method taking $h=0.1$ and $\Delta t=h / 10)$, we observed that only the expected numerical dispersive tail was observed; the latter had an amplitude of $O\left(10^{-6}\right)$ and diminished fast as $h$ was decreased. 
The wavelet also appears after numerical peakon interactions effected by other type of discretizations. For example, the pseudospectral-RK4 method with $N=16,384, \Delta t=5 \times 10^{-3}$ gives for the analogous experiment shown in Figure 5. at $t=200$ the profile given in Figure 6, very similar to the one of Figure 5(f). The wavelet and the initial oscillations diminish slowly as $N$ is increased and $\Delta t$ is decreased. The fact that the numerical interaction of peakons with spectral methods give small errors that diminish slowly as the mesh is refined was already observed in 31. Here we have pointed out that the small errors are of

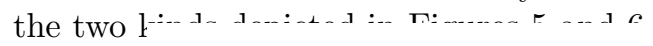
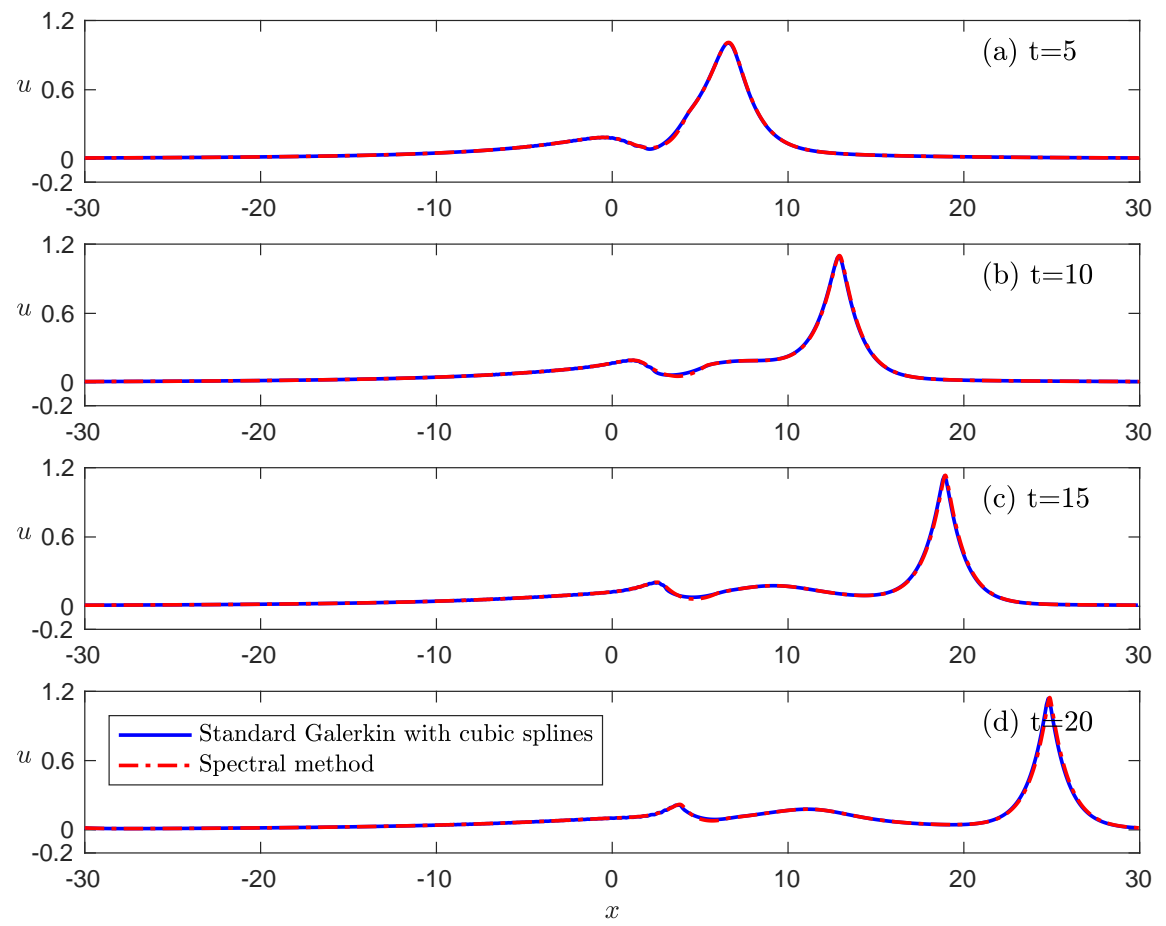

Figure 7. Evolution of RCH with initial profile (6.10), standard Galerkin method with cubic splines and pseudospectral-RK4 scheme

(iii) We finally present results obtained by applying our numerical methods to two evolution problems for $\mathrm{RCH}$ that have been proposed in the literature as examples of peakon generation from continuous initial profiles that have discontinuous derivatives. The first, [27, 46, 10, corresponds to the initial value

$$
u_{0}(x)=\frac{10}{(3+|x|)^{2}}, \quad x \in[-30,30],
$$

which we integrated up to $t=20$ by our fully discrete standard Galerkin method with cubic splines using $h=0.1, \Delta t=0.001$, cf. Figure 7 . Superimposed on the spline graphs of Figure 7 are the corresponding obtained by the usual pseudospectral-RK4 discretization of RCH with $N=4096, \Delta t=0.01$. (For smaller values of $N$ the pseudospectral method had a noticeable phase error by $t=20$.)

The second initial profile, [2, 46, corresponds to the plateau function given by

$$
u_{0}(x)=\left\{\begin{array}{ll}
c e^{x+5} & , \quad x \leq-5 \\
c & , \quad|x| \leq 5 \\
c e^{-x+5} & , \quad x \geq 5
\end{array} \quad x \in[-40,40], \quad c=0.6,\right.
$$

and produces the evolution shown up to $t=36$ in Figure 8. For the fully discrete stasdard Galerkin scheme with cubic splines we took $h=0.1$ and $\Delta t=0.01$. Superimposed are shown the corresponding profiles generated by the usual pseudospectral-RK4 scheme for $N=8192, \Delta t=0.001$. For smaller values of $N$ the scheme exhibited phase and amplitude errors in approximating the larger emerging peakon. In both test problems the Galerkin method gave very accurate results. 

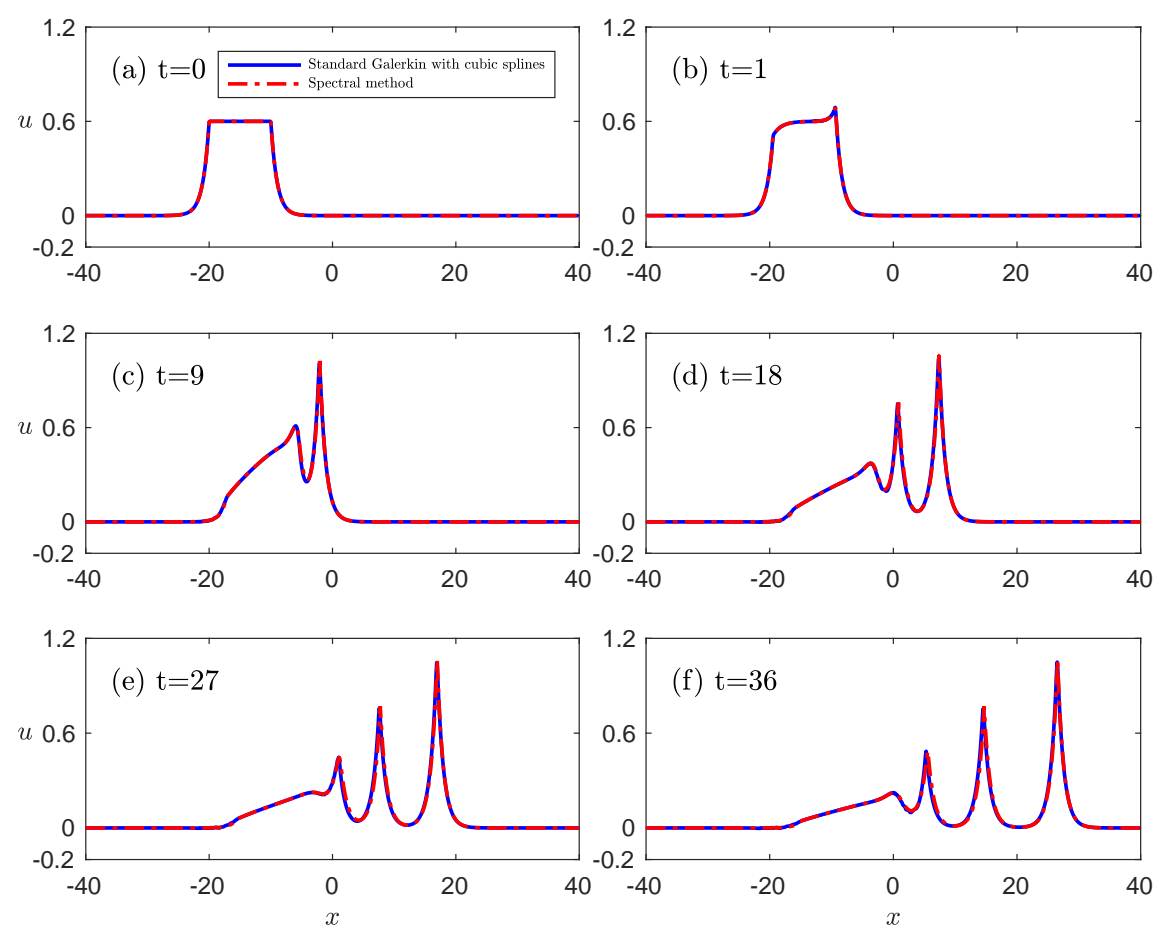

FiguRE 8. Evolution of RCH with initial profile (6.11), Standard Galerkin method with cubic splines and pseudospectral-RK4

\section{REFERENCES}

[1] D.C. Antonopoulos, V.A. Dougalis, and D. Mitsotakis. Numerical solution of Boussinesq systems of the Bona-Smith family. Appl. Numer. Math., 60:314-336, 2010.

[2] R. Artebrant and H.J. Schroll. Numerical simulation of Camassa-Holm peakons by adaptive upwinding. Appl. Numer. Math., 56:695-711, 2006.

[3] T.B. Benjamin, J.L. Bona, and J.J. Mahony. Model equations for long waves in nonlinear dispersive systems. Phil. Trans. R. Soc. Lond. A, 272:47-78, 1972.

[4] J.L. Bona, V.A. Dougalis, O.A. Karakashian, and W.R. McKinney. Conservative, high-order numerical schemes for the generalized Korteweg-de Vries equation. Phil. Trans. R. Soc. Lond. A, 351:107-164, 1995.

[5] J.L. Bona, W.G. Pritchard, and L.R. Scott. Numerical schemes for a model for nonlinear dispersive waves. J. Comp. Phys., 60:167-186, 1985.

[6] A. Bressan and A. Constantin. Global conservative solutions of the Camassa-Holm equation. Arch. Rational Mech. Anal., 183:215-239, 2007.

[7] R. Camassa and D.D. Holm. An integrable shallow water equation with peaked solitons. Phys. Rev. Lett., 71:1661-1664, 1993.

[8] R. Camassa, D.D. Holm, and J.M. Hyman. A New Integrable Shallow Water Equation. Adv. Appl. Mech., 31:1-33, 1994.

[9] A. Chertock, J.-G. Liu, and T. Pendleton. Convergence of a particle method and global weak solutions of a family of evolutionary PDEs. SIAM J. Numer. Anal., 50:1-21, 2012.

[10] A. Chertock, J.-G. Liu, and T. Pendleton. Elastic collisions among peakon solutions for the Camassa-Holm equation. Appl. Num. Math., 93:30-46, 2015.

[11] G.M. Coclite, K.H. Karlsen, and N.H. Risebro. A convergent finite difference scheme for the Camassa-Holm equation with general $h^{1}$ initial data. SIAM J. Numer. Anal., 46:1554-1579, 2008.

[12] A. Constanin and J. Lenells. On the inverse scattering approach to the Camassa-Holm equation. J. Nonlinear Math. Phys, 10:252-255, 2003.

[13] A. Constantin. On the Cauchy problem for the periodic Camassa-Holm equation. J. Differential Equations, 141:218-235, 1997.

[14] A. Constantin. On the scattering problem for the Camassa-Holm equation. Proc. R. Soc. London A, 457:953-970, 2001.

[15] A. Constantin and J. Escher. Global existence and blow-up for a shallow water equation. Annali Scuola Norm. Sup. Pisa, 26:303-328, 1998.

[16] A. Constantin and J. Escher. Wave breaking for nonlinear nonlocal shallow water equations. Acta Math., 181:229-243, 1998. 
[17] A. Constantin and D. Lannes. The hydrodynamical relevance of the Camassa-Holm and Degasperis-Procesi equations. Arch. Rational Mech. Anal., 192:165-186, 2009.

[18] A. Constantin and L. Molinet. Orbital stability of solitary waves for a shallow water equation. Physica D, 57:75-89, 2001.

[19] A. Constantin and W. Strauss. Stability of peakons. Comm. Pure Appl. Math, 53:603-610, 2000.

[20] A. Constantin and W. Strauss. Stability of the Camassa-Holm solitons. J. Nonlinear Sci., 12:415-422, 2002.

[21] C. De Boor and G.J. Fix. Spline approximation by quasiinterpolants. J. Approx. Theory, 8:19-45, 1973.

[22] Jr. Douglas, J., T. Dupont, and J.B. Wahlbin. Optimal $l^{\infty}$ error estimates for Galerkin approximations to solutions of two-point boundary value problems. Math. Comp., 29:475-483, 1975.

[23] J. Escher and Z. Yin. Initial boundary value problems of the Camassa-Holm equation. Communications in Partial Differential Equations, 33:377-395, 2008.

[24] A.S. Fokas. On a class of physically important integrable equations. Physica D, 87:145-150, 1995.

[25] B Fuchssteiner. Some tricks from the symmetry-toolbox for nonlinear equations: Generalizations of the Camassa-Holm equation. Physica D, 95:229-243, 1996.

[26] B. Fuchssteiner and A.S. Fokas. Symplectic structures, their Bäcklund tranformations and hereditary symmetries. Physica $D, 4: 47-66,1981$.

[27] H. Holden and X. Raynaud. A convergent numerical scheme for the Camassa-Holm equation based on multipeakons. Discr. Contin. Dyn. Syst., 14:505-523, 2006.

[28] H. Holden and X. Raynaud. Convergence of a finite difference scheme for the Camassa-Holm equation. SIAM J. Numer. Anal., 44:1655-1680, 2006.

[29] R.S. Johnson. Camassa-Holm, Korteweg-de Vries and related models for water waves. J. Fluid Mech., 455:63-82, 2002.

[30] R.S. Johnson. On solutions of the Camassa-Holm equation. Proc. R. Soc. London A, 459:1687-1708, 2003.

[31] H. Kalisch and J. Lenells. Numerical study of traveling-wave solutions for the Camassa-Holm equation. Chaos, Solitons and Fractals, 25:287-298, 2005.

[32] H. Kalisch and X. Raynaud. Convergence of a spectral projection of the Camassa-Holm equation. Num. methods for PDE's, 22:1197-1215, 2006.

[33] K.H. Kwek, H. Gao, W. Zhang, and C. Qu. An initial boundary value problem of Camassa-Holm equation. J. Math. Phys., 41:8279-8285, 2000 .

[34] J. Lenells. Traveling wave solutions of the Camassa-Holm equation. J. Differential Equations, 217:393-430, 2005.

[35] Y.A. Li and P.J. Olver. Well-posedness and blow-up solutions for an integrable nonlinearly dispersive model wave equation. J. Differential Equations, 162:27-63, 2000.

[36] H. Liu and Y. Xing. An invariant preserving discontinuous Galerkin method for the Camassa-Holm Equation. SIAM J. Sci. Comput., 38:A1919-A1934, 2016.

[37] L. Molinet. On well-posedness results for Camassa-Holm equation on the line: a survey. J. Nonlinear Math. Phys., 11:521533, 2004

[38] A. Parker. On the Camassa-Holm equation and a direct method of solution I. Bilinear form and solitary waves. Proc. R. Soc. London A, 460:2929-2957, 2004.

[39] A. Parker. On the Camassa-Holm equation and a direct method of solution. II. Soliton solutions. Proc. R. Soc. London A, 461:3611-3632, 2005.

[40] A. Parker. On the Camassa-Holm equation and a direct method of solution. III. N-soliton solutions. Proc. R. Soc. London A, 461:3893-3911, 2005.

[41] A Parker. Wave dynamics for peaked solitons of the Camassa-Holm equation. Chaos, Solitons and Fractals, 35:220-237, 2008.

[42] R. Rannacher and R. Scott. Some optimal error estimates for piecewise linear finite element approximations. Math. Comp., 38:437-445, 1982.

[43] R. Schreiber. Finite element methods of high-order accuracy for singular two-point boundary value problems with nonsmooth solutions. SIAM J. Numer. Anal., 17:547-566, 1980.

[44] V. Thomée and L.B. Wahlbin. Maximum-norm stability and error estimates in Galerkin methods for parabolic equations in one space variable. Numer. Math., 41:345-371, 1983.

[45] V. Thomée and B. Wendroff. Convergence estimates for Galerkin methods for variable coefficient initial value problems. SIAM J. Numer. Anal., 11:1059-1068, 1974.

[46] Y. Xu and C.-W. Shu. A local discontinuous Galerkin method for the Camassa-Holm equation. SIAM J. Numer. Anal., 46:1998-2021, 2008. 
D.C. Antonopoulos: Mathematics Department, National and Kapodistrian University of Athens, 15784 Zographou, Greece, Intitute of Applied and Computational Mathematics, FOrTH, 70013 Heraklion, Greece

E-mail address: antonod@math.uoa.gr

V. A. Dougalis: Mathematics Department, National and Kapodistrian University of Athens, 15784 Zographou, Greece, Intitute of Applied and Computational Mathematics, FORTH, 70013 Heraklion, Greece

E-mail address: doug@math.uoa.gr

D. Mitsotakis: Victoria University of Wellington, School of Mathematics and Statistics, PO Box 600, WellingTON 6140, NEW ZEALAND

E-mail address: dimitrios.mitsotakis@vuw.ac.nz 